\title{
Harmonic Analysis and Free Field Realization of the Takiff Supergroup of GL(1|1)
}

\author{
Andrei BABICHENKO ${ }^{\dagger}$ and Thomas CREUTZIG ${ }^{\ddagger}$ \\ $\dagger$ Department of Mathematics, Weizmann Institut, Rehovot, 76100, Israel \\ E-mail: babichenkoandrei@gmail.com \\ $\ddagger$ Department of Mathematical and Statistical Sciences, University of Alberta, \\ Edmonton, Alberta T6G 2G1, Canada \\ E-mail: creutzig@ualberta.ca
}

Received May 28, 2015, in final form August 01, 2015; Published online August 06, 2015

http://dx.doi.org/10.3842/SIGMA.2015.067

\begin{abstract}
Takiff superalgebras are a family of non semi-simple Lie superalgebras that are believed to give rise to a rich structure of indecomposable representations of associated conformal field theories. We consider the Takiff superalgebra of $\mathfrak{g l}(1 \mid 1)$, especially we perform harmonic analysis for the corresponding supergroup. We find that every simple module appears as submodule of an infinite-dimensional indecomposable but reducible module. We lift our results to two free field realizations for the corresponding conformal field theory and construct some modules.
\end{abstract}

Key words: logarithmic CFT; Harmonic analysis; free field realization

2010 Mathematics Subject Classification: 17B67; 17B81; 22E46; 81R10; 81T40

\section{Introduction}

Logarithmic conformal field theories carry this name as correlation functions sometimes have logarithmic singularities. They are playing an essential role in different physical problems ranging from string theory, especially on supergroup target spaces $[2,5,6,7,28,34,35,39,40,43,45]$ to different condensed matter and statistical mechanics problems [24, 25, 26, 33, 37, 38, 41, 42, 47]. Many of these statistical mechanics problems are described by logrithmic CFTs based on Lie superalgebras, as, e.g., supersymmetric disordered systems [29, 50]. For reviews on logarithmic CFT, see [13, 21, 23].

The presence of logarithmic singularities is tightly connected to the non semi-simple action of the chiral algebra on some of its modules. In some models [3] of non chiral conformal theory related to string theory on $\mathrm{AdS}_{3} \times S^{3}$ non semi-simplicity appears on the level of the assumed symmetry, and not only the representation level. In [4], motivated by this fact, different chiral conformal field theories were considered, where non semi-simplicity appears on the level of symmetry algebra itself. These are conformal field theories based on Takiff (super)algebras, e.g., algebras that are non semi-simple extensions of simple Lie (super)algebras by its adjoint representation. Detailed algebraic structure of its representation theory was then investigated.

In the mathematics literature, this type of non-semisimple Lie superalgebras were introduced by Takiff [46], though not in the super setting, but as part of an investigation of invariant polynomial rings. These algebras have since been considered in a slightly generalised form under the names generalised Takiff algebras $[27,49]$ in which a semisimple Lie algebra is tensored with a polynomial ring in a nilpotent formal variable $t$, and truncated current algebras $[48,49]$ in which one does the same to an affine Kac-Moody algebra. The algebras considered in [4] correspond to taking $t^{2}=0$, as in Takiff's original paper, and they were named Takiff superalgebras. These 
algebras were mainly considered algebraically from the representation theory point of view, in their chiral sector. On the other hand, it is well known that potential additional algebraic structures appear for Wess-Zumino-Novikov-Witten (WZNW) theories when representations are subjected to both chiral and anti chiral action of the group. In the present work, we initiate the study of the WZNW theory for the Takiff Lie supergroup GL(1|1). In [19, 20, 36], WZNW theories of non-reductive Lie groups have been studied and our notion of Takiff superalgebra is what the authors of [20] there call a double extensions.

Wess-Zumino-Novikov-Witten theories of non-compact Lie groups and Lie supergroups provide a rich source of logarithmic conformal field theories. Usually, one considers such theories based on a semi-simple Lie (super)group or closely related supergroups as GL $(n \mid n) .{ }^{1}$ Experience shows that in such theories most modules are actually completely reducible and only a few nongeneric modules are indecomposable but reducible. It is an interesting situation if already the Lie (super)algebra/Lie (super)group on which the conformal field theory is based is itself non semi-simple, as, e.g., for Takiff (super)groups. In [4], the first author and David Ridout started to study such algebras from a conformal field theory perspective. Here, we'd like to continue their work with the aim of studying an example of a conformal field theory based on a Takiff superalgebra in detail. We believe that the case of $\widehat{\mathfrak{g l}(1 \mid 1)}$ (the Takiff superalgebra of the Lie superalgebra $\mathfrak{g l}(1 \mid 1))$ is both treatable and instructive, so that we decided to first focus on this case. The chiral algebra of a WZNW model of a Lie group at level $k$ is the simple affine vertex operator algebra of its Lie algebra $\mathfrak{g}$ at level $k$. Frenkel and Zhu [22] have proven that there is a one-to-one correspondence of simple representations of this vertex algebra and simple representations of a quotient of the universal envelopping algebra of the Lie algebra $\mathfrak{g}$. If the vacuum Verma module of the affine vertex operator algebra is simple then there is actually a one-to-one correspondence to the simple objects of the universal envelopping algebra. As in the case of $\mathfrak{g l}(1 \mid 1)$, the vacuum Verma module of the vertex algebra of $\overline{\mathfrak{g l}(1 \mid 1)}$ is simple for non-vanishing levels [4]. So, we expect that representations of $\overline{\mathfrak{g l}(1 \mid 1)}$ can teach us quite a bit about the conformal field theory. Very natural modules of the Lie superalgebra are functions on the Lie supergroup which are studied using harmonic analysis (often called mini superspace analysis in the CFT literature) on the Lie supergroup. This is actually a very general experience in WZNW theories based on Lie supergroups. The key strategy is to understand a problem of the finite-dimensional Lie superalgebra, to lift this understanding to the full conformal field theory, usually to a free field realization, and then to use the new understanding to derive interesting statements concerning the CFT. This strategy has been initiated by Hubert Saleur, Volker Schomerus and Thomas Quella a decade ago. It has been used extensively by various people, including the second author. For example, the key starting ingredient of the bulk theories of $\mathrm{GL}(1 \mid 1)$ [45], $\mathrm{SU}(2 \mid 1)$ [44], $\mathrm{PSU}(1,1 \mid 2)$ [28] as well as the more general case [39] has been the harmonic analysis, and then lifting the findings to an appropriate free field realization. These free field realizations in turn were very useful in computing correlation functions [45], proving dualities to super Liouville field theories [11,30] and finding relations to CFTs with $\mathcal{N}=(2,2)$ world-sheet superconformal symmetry [17]. Similarly also boundary theories based on GL(1|1) [12, 18] and $\operatorname{OSP}(1 \mid 2)$ [10] have been studied by first performing an appropriate harmonic analysis. The difference to the bulk theory is, that instead of studying the action of both left- and right-invariant vector fields one investigates a twisted adjoint action. Boundary states are then lifts of distributions localized on twisted superconjugacy classes [9, 12].

The by far best understood WZNW theory of a Lie supergroup is the one of GL(1|1). It has been first studied by Rozansky and Saleur a while ago [43]. Then as mentioned, Schomerus and

\footnotetext{
${ }^{1}$ Even though $\mathfrak{g l}(n \mid n)$ is not semi-simple its representation theory is similar to the one of the simple Lie superalgebra $\mathfrak{p s l}(n \mid n)$. However, already in the case of $\mathfrak{g l}(1 \mid 1)$ representations of the Takiff superalgebra have much more involved indecomposability structure than the non-Takiff superalgebra [4].
} 
Saleur, were able to understand spectrum and correlation functions using harmonic analysis and free field realization [45]. As the next step boundary states were constructed [12]. Studying the twisted adjoint action of invariant vector fields on functions on the supergroup allowed one to find the boundary states explicitely. Boundary and bulk-boundary correlation functions could also be computed using a similar free field realization as in the bulk case [18]. The indecomposability structure of modules is not directly visible in the free field realization used in the just mentioned works. It turns out that there is another free field realization using symplectic fermions that makes this structure much clearer [16, 32]. Using then the work of Kausch on symplectic fermions [31] most of the previous results could be obtained fairly directly [16]. This conformal field theory can also be studied from a more algebraic perspective, especially many extended algebras like $\mathfrak{s l}(2 \mid 1)$ at levels $-1 / 2$ and 1 , can be constructed and studied $[1,14,15]$. In summary, the GL(1|1) WZNW theory has been thoroughly studied and has revealed a rich structure. We believe that a similar (but much more complex) story holds for its Takiff superalgebra. In [4], representations of both finite-dimensional and affine Takiff superalgebra of $\mathfrak{g l}(1 \mid 1)$ have been studied and a Verlinde fusion ring has been computed. We now would like to proceed to the next step, and ask which representations appear in the full bulk CFT, and wether we can realize them using free fields. Our main result is a thorough harmonic analysis of the Takiff supergroup. We find that functions split into three classes, called typical, semitypical and atypical. All of them appear in the harmonic analysis as submodule of infinitedimensional indecomposable but reducible modules. The reason for this is a special element $\tilde{N}$ of the Lie superalgebra, that already acts non-semi-simple in the adjoint representation. It forces us to allow for a larger space of functions. Nonetheless, in the typical case there is still a decomposition into tensor products of modules for the action of left- and right-invariant vector fields somehow similar as one is used from the Peter-Weyl theorem for compact Lie groups. Both semitypical and especially atypical modules have a rather complicated behaviour under the left-right action. We visualize the structure of these modules in various pictures and exact sequences. Further, already the simple submodules of the typical modules posses Jordan cells of size three under the action of the Laplacian (as expected from [4]). The harmonic analysis tells us nicely how to construct a free field realization including screening charges. As in the case of the GL(1|1) WZNW theory, typical modules can be constructed easily using this free field realization. Again, in analogy to the GL(1|1) WZNW theory we find a second free field realization using symplectic fermions which allows us to also construct semitypical and atypical modules. This puts us now in a position to study (in the near future) these modules further, to ask questions about correlation functions, operator product algebra and extended algebras.

This work is organized as follows: Section 2 is the main part of this paper. We first compute basic objects on the Takiff supergroup, namely invariant vector fields, invariant measure and Laplacians. Then we compute the action of invariant vector fields. The typical case can be performed rather directly, while the structure in the semitypical and atypical cases is very rich and first studying the action of various subalgebras turns out to structure the problem nicely. Section 3 then applies our findings to construct a free field realization including screening charges. But we also add another free field realization using symplectic fermions. Finally, we conclude with describing the expected future use of our findings.

\section{Harmonic analysis}

We start from definition of Takiff superalgebra $\widetilde{\mathrm{GL}(1 \mid 1)}$, and briefly describe its highest weight representations. The main part of this section is devoted to harmonic analysis for GL(1|1) supergroup. The procedure for this follows ideas established in various works on GL(1|1) (see mainly [45]) and goes as follows: 
1) consider a supergroup element $g$ according to a triangular decomposition;

2) compute left- and right-invariant vector fields;

3) compute the invariant measure;

4) compute the Laplacians;

5) decompose functions with respect to the combined left-right action of invariant vector fields.

\subsection{Takiff superalgebra $\widetilde{\mathfrak{g l}(1 \mid 1)}$ and its highest weight representations}

Takiff Lie superalgebra $\widetilde{\mathfrak{g l}(1 \mid 1)}$ was introduced in [4] as an extention of the superalgebra $\mathfrak{g l}(1 \mid 1)$ with Grassmann even generator $N$, Grassmann odd generators $\psi^{ \pm}$and central element $E$

$$
\left[N, \psi^{ \pm}\right]= \pm \psi^{ \pm}, \quad\left\{\psi^{+}, \psi^{-}\right\}=E
$$

by a set of their Takiff partners $\tilde{N}, \tilde{\psi}^{ \pm}, \tilde{E}$ with commutation relations

$$
\left[N, \tilde{\psi}^{ \pm}\right]=\left[\tilde{N}, \psi^{ \pm}\right]= \pm \tilde{\psi}^{ \pm}, \quad\left\{\tilde{\psi}^{+}, \psi^{-}\right\}=\left\{\psi^{+}, \tilde{\psi}^{-}\right\}=\tilde{E}
$$

and the remaining (anti)commutators vanish.

As one can see the adjoint action of $N, E, \tilde{E}$ is diagonalised in the chosen basis of generators, but the adjoint action of $\widetilde{N}$ acts non semisimply on adjoint module. The obvious triangular decomposition

$$
\widetilde{\mathfrak{g l}(1 \mid 1)}=\operatorname{span}\left\{\psi^{-}, \tilde{\psi}^{-}\right\} \oplus \operatorname{span}\{N, E, \tilde{N}, \tilde{E}\} \oplus \operatorname{span}\left\{\psi^{+}, \widetilde{\psi}^{+}\right\}
$$

gives rise to highest weight modules: the highest weight is defined as an eigenvector of $N$, $E, \tilde{N}, \tilde{E}$ with eigenvalues $n, e, \tilde{n}, \tilde{e}$ correspondingly, which is annihilated by the action of raising generators $\psi^{+}, \tilde{\psi}^{+}$. The highest weight Verma module is defined as a free action of lowering generators $\psi^{-}, \tilde{\psi}^{-}$on the highest weight. Since $\psi^{-}, \tilde{\psi}^{-}$are Grassmann odd and anticommute, the Verma module is four-dimensional. All the states of the Verma module with highest weight $|v\rangle$ are eigenstates of $\tilde{N}$ except for $\psi^{-}|v\rangle$ on which it acts non semisimply: $\tilde{N} \psi^{-}|v\rangle=\tilde{n} \psi^{-}|v\rangle-\tilde{\psi}^{-}|v\rangle$. As it was shown in [4], there are three possibilities for irreducible quotients of Verma module. If $e=\tilde{e}=0$, the irreducible quotient is one-dimensional and was called atypical, if $\tilde{e}=0$, but $e \neq 0$, it is two-dimensional and was called semitypical, and if $\tilde{e} \neq 0$, the Verma module is irreducible. In a similar way one can define lowest weight Verma modules and their irreducible quotients.

There are two linearly independent quadratic Casimir operators in the universal enveloping algebra of $\widehat{\mathfrak{g l}(1 \mid 1)}$ (modulo polynomials in central elements) which can be chosen as

$$
Q_{1}=N \tilde{E}+\tilde{N} E+\psi^{-} \tilde{\psi}^{+}+\tilde{\psi}^{-} \psi^{+}, \quad Q_{2}=\tilde{N} \tilde{E}+\tilde{\psi}^{-} \tilde{\psi}^{+} .
$$

They act on the highest weight Verma module as multiplication by $n \tilde{e}+\tilde{n} e, \tilde{n} \tilde{e}$ respectively.

An important object for affinization of Lie (super)algebras is a non-degenerate symmetric bilinear form $\kappa$ on the algebra. Among different possibilities, we choose the lifting of the standard bilinear form $\kappa_{0}(X, Y)$ of $\mathfrak{g l}(1 \mid 1)$ (defined as supertrace $\operatorname{str}(X Y)$ in the defining representation of it) as follows

$$
\tilde{\kappa}(\tilde{X}, Y)=\kappa_{0}(X, Y), \quad \tilde{\kappa}(\tilde{X}, \tilde{Y})=\tilde{\kappa}(X, Y)=0 .
$$




\subsection{Invariant vector fields}

We choose a Lie supergroup parameterization according to above triangular decomposition

$$
g=e^{i \theta_{+} \psi^{+}+i \tilde{\theta}_{+} \tilde{\psi}^{+}} e^{i x E+i y N+i \tilde{x} \tilde{E}+i \tilde{y} \tilde{N}} e^{i \theta_{-} \psi^{-}+i \tilde{\theta}_{-} \tilde{\psi}^{-}} .
$$

Invariant vector fields on Lie supergroups have to be treated with attention to signs to (see [8]). We define them by

$$
R_{+} g=\psi^{+} g, \quad \tilde{R}_{+} g=\tilde{\psi}^{+} g, \quad R_{X} g=-X g
$$

for all $X$ in the complement of the $\psi^{+}, \tilde{\psi}^{+}$. This definition guarantees that the invariant vector fields obey the relations of $\widetilde{\mathfrak{g l}(1 \mid 1)}$

$$
R_{X} R_{Y}-(-1)^{|X||Y|} R_{Y} R_{X}=R_{[X, Y]} .
$$

Then a computation reveals that

$$
\begin{aligned}
& \psi^{+} g=-i \frac{d}{d \theta_{+}} g, \quad \tilde{\psi}^{+} g=-i \frac{d}{d \tilde{\theta}_{+}} g, \quad E g=-i \frac{d}{d x} g, \quad \tilde{E} g=-i \frac{d}{d \tilde{x}} g \\
& N g=-i \frac{d}{d y} g+i \theta_{+} \psi^{+} g+i \tilde{\theta}_{+} \tilde{\psi}^{+} g, \quad \tilde{N} g=-i \frac{d}{d \tilde{y}} g+i \theta_{+} \tilde{\psi}^{+} g \\
& \psi^{-} g=-i e^{i y} \frac{d}{d \theta_{-}} g+i \tilde{y} \tilde{\psi}^{-} g-i \theta_{+} E g-i \tilde{\theta}_{+} \tilde{E} g-\tilde{y} \theta_{+} \tilde{E} g, \quad \tilde{\psi}^{-} g=-i e^{i y} \frac{d}{d \tilde{\theta}_{-}} g-i \theta_{+} \tilde{E} g .
\end{aligned}
$$

So that

$$
\begin{aligned}
& R_{+}=-i \frac{d}{d \theta_{+}}, \quad \tilde{R}_{+}=-i \frac{d}{d \tilde{\theta}_{+}}, \quad R_{E}=i \frac{d}{d x}, \quad \tilde{R}_{E}=i \frac{d}{d \tilde{x}}, \\
& R_{N}=i \frac{d}{d y}-\theta_{+} \frac{d}{d \theta_{+}}-\tilde{\theta}_{+} \frac{d}{d \tilde{\theta}_{+}}, \quad \tilde{R}_{N}=i \frac{d}{d \tilde{y}}-\theta_{+} \frac{d}{d \tilde{\theta}_{+}}, \\
& R_{-}=i e^{i y}\left(\frac{d}{d \theta_{-}}+i \tilde{y} \frac{d}{d \tilde{\theta}_{-}}\right)-\theta_{+} \frac{d}{d x}-\tilde{\theta}_{+} \frac{d}{d \tilde{x}}, \quad \tilde{R}_{-}=i e^{i y} \frac{d}{d \tilde{\theta}_{-}}-\theta_{+} \frac{d}{d \tilde{x}} .
\end{aligned}
$$

In the same way for the left-action we require $L_{-} g=-g \psi^{-}, \tilde{L}_{-} g=-g \tilde{\psi}^{-}$and $L_{X} g=X g$ for all $X$ in the complement of the $\psi^{-}, \tilde{\psi}^{-}$. Then one gets

$$
\begin{aligned}
& g \psi^{-}=-i \frac{d}{d \theta_{-}} g, \quad g \tilde{\psi}^{-}=-i \frac{d}{d \tilde{\theta}_{-}} g, \quad g E=-i \frac{d}{d x} g, \quad g \tilde{E}=-i \frac{d}{d \tilde{x}} g, \\
& g N=-i \frac{d}{d y} g+\theta_{-} \frac{d}{d \theta_{-}} g+\tilde{\theta}_{-} \frac{d}{d \tilde{\theta}_{-}} g, \quad g \tilde{N}=-i \frac{d}{d \tilde{y}} g+\theta_{-} \frac{d}{d \tilde{\theta}_{-}} g, \\
& g \psi^{+}=-i e^{i y}\left(\frac{d}{d \theta_{+}}+i \tilde{y} \frac{d}{d \tilde{\theta}_{+}}\right) g-\theta_{-} \frac{d}{d x} g-\tilde{\theta}_{-} \frac{d}{d \tilde{x}} g, \quad g \tilde{\psi}^{+}=-i e^{i y} \frac{d}{d \tilde{\theta}_{+}} g-\theta_{-} \frac{d}{d \tilde{x}} g .
\end{aligned}
$$

So that for the left-action we have

$$
\begin{aligned}
& L_{-}=i \frac{d}{d \theta_{-}}, \quad \tilde{L}_{-}=i \frac{d}{d \tilde{\theta}_{-}}, \quad L_{E}=-i \frac{d}{d x}, \quad \tilde{L}_{E}=-i \frac{d}{d \tilde{x}}, \\
& L_{N}=-i \frac{d}{d y}+\theta_{-} \frac{d}{d \theta_{-}}+\tilde{\theta}_{-} \frac{d}{d \tilde{\theta}_{-}}, \quad \tilde{L}_{N}=-i \frac{d}{d \tilde{y}}+\theta_{-} \frac{d}{d \tilde{\theta}_{-}}, \\
& L_{+}=-i e^{i y}\left(\frac{d}{d \theta_{+}}+i \tilde{y} \frac{d}{d \tilde{\theta}_{+}}\right)-\theta_{-} \frac{d}{d x}-\tilde{\theta}_{-} \frac{d}{d \tilde{x}}, \quad \tilde{L}_{+}=-i e^{i y} \frac{d}{d \tilde{\theta}_{+}}-\theta_{-} \frac{d}{d \tilde{x}} .
\end{aligned}
$$




\subsection{The Haar measure}

The left-invariant Maurer-Cartan form is

$$
\omega(g)=g^{-1} d g
$$

and the right-invariant Maurer-Cartan form is $\omega\left(g^{-1}\right)$. Either one can be taken to compute the Haar measure and we will use the right-invariant one

$$
\omega\left(g^{-1}\right)=-d g g^{-1}=-\left(\frac{d}{d \theta_{+}} g\right) g^{-1} d \theta_{+}-\left(\frac{d}{d \tilde{\theta}_{+}} g\right) g^{-1} d \tilde{\theta}_{+}-\cdots
$$

This is a Lie superalgebra valued one form, that is it can be written as

$$
\begin{aligned}
\omega\left(g^{-1}\right)= & \omega\left(\psi^{+}\right) \psi^{+}+\omega\left(\tilde{\psi}^{+}\right) \tilde{\psi}^{+}+\omega(E) E+\omega(\tilde{E}) \tilde{E}+\omega(N) N+\omega(\tilde{N}) \tilde{N} \\
& +\omega\left(\psi^{-}\right) \psi^{-}+\omega\left(\tilde{\psi}^{-}\right) \tilde{\psi}^{-} .
\end{aligned}
$$

The components are the dual one-forms in our basis. Using (2.2) they can be easily extracted

$$
\begin{array}{ll}
\omega\left(\psi^{+}\right)=-i d \theta_{+}-\theta_{+} d y, & \omega\left(\tilde{\psi}^{+}\right)=-i d \tilde{\theta}_{+}-\tilde{\theta}_{+} d y-\theta_{+} d \tilde{y}, \\
\omega(E)=-i d x+e^{-i y} \theta_{+} d \theta_{-}, & \omega(\tilde{E})=-i d \tilde{x}+e^{-i y}\left(\tilde{\theta}_{+}-i \tilde{y} \theta_{+}\right) d \theta_{-}+e^{-i y} \theta_{+} d \tilde{\theta}_{-}, \\
\omega(N)=-i d y, & \omega(\tilde{N})=-i d \tilde{y}, \\
\omega\left(\psi^{-}\right)=-i e^{-i y} d \theta_{-}, & \omega\left(\tilde{\psi}^{-}\right)=-i e^{-i y} d \tilde{\theta}_{-}-e^{-i y} \tilde{y} d \theta_{-} .
\end{array}
$$

The right-invariant measure is the wedge product of these dual one forms

$$
\begin{aligned}
\mu\left(g^{-1}\right) & =\omega\left(\psi^{+}\right) \wedge \omega\left(\tilde{\psi}^{+}\right) \wedge \omega(E) \wedge \omega(\tilde{E}) \wedge \omega(N) \wedge \omega(\tilde{N}) \wedge \omega\left(\psi^{-}\right) \wedge \omega\left(\tilde{\psi}^{-}\right) \\
& =e^{-2 i y} d \theta_{+} \wedge d \tilde{\theta}_{+} \wedge d x \wedge d \tilde{x} \wedge d y \wedge d \tilde{y} \wedge d \theta_{-} \wedge d \tilde{\theta}_{-} .
\end{aligned}
$$

Here, we used graded anti-symmetry of the wedge product as well as integration with respect to an odd variable is the same as taking the derivative, but the double derivative of an odd variable vanishes, in other words we have used $d \theta \wedge d \theta=0$.

\subsection{The Laplace operators}

In [4] two Casimir operators of $\widehat{\mathfrak{g l}(1 \mid 1)}$ were given. We change one of them by the central element $\tilde{E}$. They are then

$$
Q_{1}=N \tilde{E}+\tilde{N} E+\psi^{-} \tilde{\psi}^{+}+\tilde{\psi}^{-} \psi^{+}-\tilde{E}, \quad Q_{2}=\tilde{N} \tilde{E}+\tilde{\psi}^{-} \tilde{\psi}^{+}
$$

We compute that

$$
\begin{aligned}
& \Delta_{1}:=Q_{1}^{\mathrm{L}}=Q_{1}^{\mathrm{R}}=-\frac{d}{d y} \frac{d}{d \tilde{x}}-i \frac{d}{d \tilde{x}}-\frac{d}{d \tilde{y}} \frac{d}{d x}+e^{i y}\left(\frac{d}{d \theta_{-}} \frac{d}{d \tilde{\theta}_{+}}+\frac{d}{d \tilde{\theta}_{-}} \frac{d}{d \theta_{+}}+i \tilde{y} \frac{d}{d \tilde{\theta}_{-}} \frac{d}{d \tilde{\theta}_{+}}\right), \\
& \Delta_{2}:=Q_{2}^{\mathrm{L}}=Q_{2}^{\mathrm{R}}=-\frac{d}{d \tilde{y}} \frac{d}{d \tilde{x}}+e^{i y} \frac{d}{d \tilde{\theta}_{-}} \frac{d}{d \tilde{\theta}_{+}} .
\end{aligned}
$$

We call these operators $\Delta$ as they are the Laplace operators on this supergroup. This finishes our preparations, and we can turn to decomposing functions with respect to the left-right action of invariant vector fields. 


\subsection{Decomposing functions}

Before we start decomposing functions, let us observe how left and right action are related,

Proposition 2.1. The change of coordinates

$$
\beta: \theta_{ \pm} \mapsto-\theta_{\mp}, \quad \tilde{\theta}_{ \pm} \mapsto-\tilde{\theta}_{\mp}, \quad x \mapsto-x, \quad \tilde{x} \mapsto-\tilde{x}, \quad y \mapsto y, \quad \tilde{y} \mapsto \tilde{y}
$$

relates the action of left- and right-invariant vector fields as

$$
\begin{aligned}
& R_{ \pm} \mapsto L_{\mp}, \quad \tilde{R}_{ \pm} \mapsto \tilde{L}_{\mp}, \quad R_{E} \mapsto L_{E}, \quad \tilde{R}_{E} \mapsto \tilde{L}_{E}, \\
& R_{N} \mapsto-L_{N}, \quad \tilde{R}_{N} \mapsto-\tilde{L}_{N} .
\end{aligned}
$$

It will turn out that there are three-types of modules appearing in the decomposition. We will call these modules typical, semi-typical and atypical as in [4].

\subsubsection{Typical modules}

Let

$$
f_{e, n, \tilde{e}, \tilde{n}}(x, \tilde{x}, y, \tilde{y})=\exp (i x e+i \tilde{x} \tilde{e}-i y n-i \tilde{n} \tilde{y}),
$$

then the crucial lemma is:

Lemma 2.2. Define the matrix

$$
M=\left(\begin{array}{cccc}
1 & \theta_{-} & \tilde{\theta}_{-} & \theta_{-} \tilde{\theta}_{-} \\
\theta_{+} & \theta_{+} \theta_{-} & \theta_{+} \tilde{\theta}_{-} & \theta_{+} \theta_{-} \tilde{\theta}_{-} \\
\tilde{\theta}_{+} & \tilde{\theta}_{+} \theta_{-} & \tilde{\theta}_{+} \tilde{\theta}_{-} & \tilde{\theta}_{+} \theta_{-} \tilde{\theta}_{-} \\
\theta_{+} \tilde{\theta}_{+} & \theta_{+} \tilde{\theta}_{+} \theta_{-} & \theta_{+} \tilde{\theta}_{+} \tilde{\theta}_{-} & \theta_{+} \tilde{\theta}_{+} \theta_{-} \tilde{\theta}_{-}
\end{array}\right)
$$

and the second-order differential operator

$$
\mathcal{D}_{e, \tilde{e}}=\frac{d}{d \tilde{\theta}_{-}} \frac{d}{d \theta_{+}}+\frac{d}{d \theta_{-}} \frac{d}{d \tilde{\theta}_{+}}+\left(i \tilde{y}-\frac{e}{\tilde{e}}\right) \frac{d}{d \tilde{\theta}_{-}} \frac{d}{d \tilde{\theta}_{+}} .
$$

Let $\tilde{e} \neq 0$, then each row of the matrix

$$
V_{e, n, \tilde{e}, \tilde{n}}=f_{e, n, \tilde{e}, \tilde{n}} \exp \left(\frac{e^{i y}}{\tilde{e}} \mathcal{D}_{e, \tilde{e}}\right) M
$$

carries the irreducible highest-weight representation of the left-regular action of highest-weight $(e, \tilde{e},-n+2,-\tilde{n})$. Each column transforms in the irreducible highest-weight representation of the right-regular action of highest-weight $(-e,-\tilde{e}, n, \tilde{n})$.

Proof. We define the function $f_{4}=\theta_{-} \tilde{\theta}_{-} f_{e, n, \tilde{e}, \tilde{n}}$, then $f_{4}$ is a highest-weight vector for both left- and right-action. The weight under the right-action is $(-e,-\tilde{e}, n, \tilde{n})$ and the weight under the left-action is $(e, \tilde{e},-n+2,-\tilde{n})$. Then under the left-action, the functions

$$
f_{1}:=f_{e, n, \tilde{e}, \tilde{n}}(x, \tilde{x}, y, \tilde{y}), \quad f_{2}:=\theta_{-} f_{e, n, \tilde{e}, \tilde{n}}, \quad f_{3}:=\tilde{\theta}_{-} f_{e, n, \tilde{e}, \tilde{n}}, \quad f_{4}:=\theta_{-} \tilde{\theta}_{-} f_{e, n, \tilde{e}, \tilde{n}},
$$

carry the four-dimensional irreducible representation of that highest-weight. Since left- and right-action commute, each of these states must be a highest-weight vector of same weight as before for the right-action. For the highest-weight $f_{1}$ the remaining three states of the typical module I are

$$
\begin{aligned}
& \tilde{\psi}_{\mathrm{I}}=\tilde{R}_{-} f_{1}=i \tilde{e} \theta_{+} f_{e, n, \tilde{e}, \tilde{n}}, \quad \psi_{\mathrm{I}}=R_{-} f_{1}=i e \theta_{+} f_{e, n, \tilde{e}, \tilde{n}}+i \tilde{e} \tilde{\theta}_{+} f_{e, n, \tilde{e}, \tilde{n}}, \\
& b_{\mathrm{I}}=R_{-} \tilde{R}_{-} f_{1}=-\tilde{e}^{2} \theta_{+} \tilde{\theta}_{+} f_{e, n, \tilde{e}, \tilde{n}} .
\end{aligned}
$$


In the same way three states completing the module II with the highest-weight $f_{2}$ are

$$
\begin{aligned}
& \tilde{\psi}_{\mathrm{II}}=\tilde{R}_{-} f_{2}=-i \tilde{e} \theta_{-} \theta_{+} f_{e, n, \tilde{e}, \tilde{n}} \\
& \psi_{\mathrm{II}}=R_{-} f_{2}=i f_{e, n-1, \tilde{e}, \tilde{n}}-i e \theta_{-} \theta_{+} f_{e, n, \tilde{e}, \tilde{n}}-i \tilde{e} \theta_{-} \tilde{\theta}_{+} f_{e, n, \tilde{e}, \tilde{n}}, \\
& b_{\mathrm{II}}=R_{-} \tilde{R}_{-} f_{2}=\tilde{e} \theta_{+} f_{e, n-1, \tilde{e}, \tilde{n}}+\tilde{e}^{2} \theta_{-} \theta_{+} \tilde{\theta}_{+} f_{e, n, \tilde{e}, \tilde{n}} .
\end{aligned}
$$

For the module III with the highest-weight $f_{3}$ we get

$$
\begin{aligned}
& \tilde{\psi}_{\mathrm{III}}=\tilde{R}_{-} f_{3}=i f_{e, n-1, \tilde{e}, \tilde{n}}+i \tilde{e} f_{e, n, \tilde{e}, \tilde{n}} \theta_{+} \tilde{\theta}_{-}, \\
& \psi_{\mathrm{III}}=R_{-} f_{3}=-\tilde{y} f_{e, n-1, \tilde{e}, \tilde{n}}-i e f_{e, n, \tilde{e}, \tilde{n}} \tilde{\theta}_{-} \theta_{+}-i \tilde{e} f_{e, n, \tilde{e}, \tilde{n}} \tilde{\theta}_{-} \tilde{\theta}_{+}, \\
& b_{\mathrm{III}}=R_{-} \tilde{R}_{-} f_{3}=-e f_{e, n-1, \tilde{e}, \tilde{n}} \theta_{+}-\tilde{e} f_{e, n-1, \tilde{e}, \tilde{n} \tilde{\theta}_{+}}+i \tilde{e} \tilde{y} f_{e, n-1, \tilde{e}, \tilde{n}} \theta_{+}+\tilde{e}^{2} f_{e, n, \tilde{e}, \tilde{n}} \tilde{\theta}_{-} \theta_{+} \tilde{\theta}_{+},
\end{aligned}
$$

and for the last module IV with highest-weight $f_{4}$

$$
\begin{aligned}
& \tilde{\psi}_{\mathrm{IV}}=\tilde{R}_{-} f_{4}=-i f_{e, n-1, \tilde{e}, \tilde{n}} \theta_{-}+i \tilde{e} f_{e, n, \tilde{e}, \tilde{n}} \theta_{-} \tilde{\theta}_{-} \theta_{+}, \\
& \psi_{\mathrm{IV}}=R_{-} f_{4}=\tilde{y} f_{e, n-1, \tilde{e}, \tilde{n}} \theta_{-}+i f_{e, n-1, \tilde{e}, \tilde{n}} \tilde{\theta}_{-}+i e f_{e, n, \tilde{e}, \tilde{n}} \theta_{-} \tilde{\theta}_{-} \theta_{+}+i \tilde{e} f_{e, n, \tilde{e}, \tilde{n}} \theta_{-} \tilde{\theta}_{-} \tilde{\theta}_{+}, \\
& b_{\mathrm{IV}}=R_{-} \tilde{R}_{-} f_{4}=f_{e, n-2, \tilde{e}, \tilde{n}}-\tilde{e} f_{e, n-1, \tilde{e}, \tilde{n}} \tilde{\theta}_{-} \theta_{+}+(i \tilde{y} \tilde{e}-e) f_{e, n-1, \tilde{e}, \tilde{n}} \theta_{-} \theta_{+}
\end{aligned}
$$

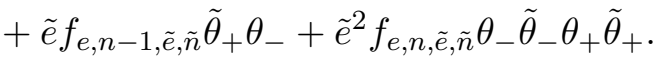

The action of $R_{N}$ is the same on all four four modules:

$$
R_{N} f_{i}=n f_{i}, \quad R_{N} \psi=(n-1) \psi, \quad R_{N} \tilde{\psi}=(n-1) \tilde{\psi}, \quad R_{N} b=(n-2) b .
$$

The action of $\tilde{R}_{N}$ is diagonal on the states $X=f_{i}, \tilde{\psi}, b: \tilde{R}_{N} X=\tilde{n} X$, but non diagonal on the states $\psi$ :

$$
\tilde{R}_{N} \psi=\tilde{n} \psi-\tilde{\psi}
$$

In summary, for $\tilde{e} \neq 0$, each row of the matrix

$$
\begin{aligned}
& f_{e, n, \tilde{e}, \tilde{n}}\left(\begin{array}{cccc}
1 & \theta_{-} & \tilde{\theta}_{-} & \theta_{-} \tilde{\theta}_{-} \\
\tilde{e} \theta_{+} & \tilde{e} \theta_{-} \theta_{+} & \tilde{e} \theta_{+} \tilde{\theta}_{-} & -\tilde{e} \theta_{-} \tilde{\theta}_{-} \theta_{+} \\
e \theta_{+}+\tilde{e} \tilde{\theta}_{+} & -e \theta_{-} \theta_{+}-\tilde{e} \theta_{-} \tilde{\theta}_{+} & e \tilde{\theta}_{-} \theta_{+}+\tilde{e} \tilde{\theta}_{-} \tilde{\theta}_{+} & e \theta_{-} \tilde{\theta}_{-} \theta_{+}+\tilde{e} \theta_{-} \tilde{\theta}_{-} \tilde{\theta}_{+} \\
\tilde{e}^{2} \theta_{+} \tilde{\theta}_{+} & \tilde{e}^{2} \theta_{-} \theta_{+} \tilde{\theta}_{+} & \tilde{e}^{2} \tilde{\theta}_{-} \theta_{+} \tilde{\theta}_{+} & \tilde{e}^{2} \theta_{-} \tilde{\theta}_{-} \theta_{+} \tilde{\theta}_{+}
\end{array}\right) \\
& +f_{e, n-1, \tilde{e}, \tilde{n}}\left(\begin{array}{cccc}
0 & 0 & 0 & 0 \\
0 & 0 & 1 & \theta_{-} \\
0 & 1 & -i \tilde{y} & -i \tilde{y} \theta_{-}+\tilde{\theta}_{-} \\
0 & \tilde{e} \theta_{+} & -e \theta_{+}-\tilde{e} \tilde{\theta}++i \tilde{e} \tilde{y} \theta_{+} & -\tilde{e} \tilde{\theta} \theta_{-} \theta_{+}+(i \tilde{y} \tilde{e}-e) \theta_{-} \theta_{+}+\tilde{e} \tilde{\theta}_{+} \theta_{-}
\end{array}\right) \\
& +f_{e, n-2, \tilde{e}, \tilde{n}}\left(\begin{array}{cccc}
0 & 0 & 0 & 0 \\
0 & 0 & 0 & 0 \\
0 & 0 & 0 & 0 \\
0 & 0 & 0 & 1
\end{array}\right)
\end{aligned}
$$

carries the irreducible highest-weight representation of the left regular action of highest-weight $(e, \tilde{e},-n+2,-\tilde{n})$. Each column transforms in the irreducible highest-weight representation of the right regular action of highest-weight $(-e,-\tilde{e}, n, \tilde{n})$. Changing basis $\psi \rightarrow \psi-\frac{e}{\tilde{e}} \tilde{\psi}$, this can be written more symmetrically,

$$
f_{e, n, \tilde{e}, \tilde{n}}\left(\begin{array}{cccc}
1 & \theta_{-} & \tilde{\theta}_{-} & \theta_{-} \tilde{\theta}_{-} \\
\theta_{+} & \theta_{+} \theta_{-} & \theta_{+} \tilde{\theta}_{-} & \theta_{+} \theta_{-} \tilde{\theta}_{-} \\
\tilde{\theta}_{+} & \tilde{\theta}_{+} \theta_{-} & \tilde{\theta}_{+} \tilde{\theta}_{-} & \tilde{\theta}_{+} \theta_{-} \tilde{\theta}_{-} \\
\theta_{+} \tilde{\theta}_{+} & \theta_{+} \tilde{\theta}_{+} \theta_{-} & \theta_{+} \tilde{\theta}_{+} \tilde{\theta}_{-} & \theta_{+} \tilde{\theta}_{+} \theta_{-} \tilde{\theta}_{-}
\end{array}\right)
$$




$$
\begin{aligned}
& +\frac{f_{e, n-1, \tilde{e}, \tilde{n}}}{\tilde{e}}\left(\begin{array}{cccc}
0 & 0 & 0 & 0 \\
0 & 0 & 1 & -\theta_{-} \\
0 & 1 & \left(i \tilde{y}-\frac{e}{\tilde{e}}\right) & -\theta_{-}\left(i \tilde{y}-\frac{e}{\tilde{e}}\right)+\tilde{\theta}_{-} \\
0 & \theta_{+} & \theta_{+}\left(i \tilde{y}-\frac{e}{\tilde{e}}\right)-\tilde{\theta}_{+} & \left(i \tilde{y}-\frac{e}{\tilde{e}}\right) \theta_{-} \theta_{+}-\tilde{\theta}_{-} \theta_{+}+\tilde{\theta}_{+} \theta_{-}
\end{array}\right) \\
& +\frac{f_{e, n-2, \tilde{e}, \tilde{n}}}{\tilde{e}^{2}}\left(\begin{array}{llll}
0 & 0 & 0 & 0 \\
0 & 0 & 0 & 0 \\
0 & 0 & 0 & 0 \\
0 & 0 & 0 & 1
\end{array}\right)
\end{aligned}
$$

so that the lemma follows.

Remark 2.3. The operator $e^{i y} \mathcal{D}_{e, \tilde{e}}$ is the semi-classical analouge of a screening charge. It is related to the Casimir operators acting on $V_{e, n, \tilde{e}, \tilde{n}}$ via

$$
\frac{1}{\tilde{e}}\left(\Delta_{1}-\frac{e}{\tilde{e}} \Delta_{2}-e^{i y} \mathcal{D}_{e, \tilde{e}}\right)=-i \frac{d}{d y}+1
$$

In other words the difference of the Casimir $\Delta_{1}-\frac{e}{\tilde{e}} \Delta_{2}$ and $e^{i y} \mathcal{D}_{e, \tilde{e}}$ is a semi-simple operator.

Finally, we define the matrices

$$
V_{e, n, \tilde{e}, \tilde{n}}^{(m)}:=\tilde{y}^{m} V_{e, n, \tilde{e}, \tilde{n}}
$$

and denote the vector space spanned by its components by $T_{e, n, \tilde{e}, \tilde{n}}^{(m)}$, so that

$$
T_{e, n, \tilde{e}, \tilde{n}}:=\bigoplus_{m=0}^{\infty} T_{e, n, \tilde{e}, \tilde{n}}^{(m)}
$$

is an infinite-dimensional module for the left-right action of $\widetilde{\mathfrak{g l}(1 \mid 1)}$. The irredicible submodule $T_{e, n, \tilde{e}, \tilde{n}}^{(0)}$ has been described in Lemma 2.2. The modules $\bigoplus_{m=0}^{N} T_{e, n, \tilde{e}, \tilde{n}}^{(m)}$ are all submodules with quotient by $\bigoplus_{m=0}^{N-1} T_{e, n, \tilde{e}, \tilde{n}}^{(m)}$ isomorphic to $T_{e, n, \tilde{e}, \tilde{n}}^{(0)}$.

\subsubsection{Semitypical modules}

The case $\tilde{e}=0$ and $e \neq 0$ is called semi-typical. Our strategy is to first decompose into irreducible $\mathfrak{g l}(1 \mid 1)$ modules. First, fix $e \neq 0, \tilde{n}$. In order to avoid too many indices we take the short-hand notation $f_{n}:=f_{e, n, 0, \tilde{n}}$. We find the following list for the left-action $L_{ \pm}$on weight modules:

$$
\begin{aligned}
& A_{1, n}=\operatorname{span}\left(f_{n}, \theta_{-} f_{n}\right), \\
& A_{2, n}=\operatorname{span}\left(\theta_{+} f_{n}, i e \theta_{-} \theta_{+} f_{n}-i f_{n-1}\right), \\
& A_{3, n}=\operatorname{span}\left(\tilde{\theta}_{+} f_{n}, i e \theta_{-} \tilde{\theta}_{+} f_{n}+\tilde{y} f_{n-1}\right), \\
& A_{4, n}=\operatorname{span}\left(\tilde{\theta}_{-} f_{n}, \theta_{-} \tilde{\theta}_{-} f_{n}\right), \\
& A_{5, n}=\operatorname{span}\left(\theta_{+} \tilde{\theta}_{+} f_{n}, i e \theta_{-} \theta_{+} \tilde{\theta}_{+} f_{n}-i \tilde{\theta}_{+} f_{n-1}-\tilde{y} \theta_{+} f_{n-1}\right), \\
& A_{6, n}=\operatorname{span}\left(\tilde{\theta}_{-} \tilde{\theta}_{+} f_{n}, i e \tilde{\theta}_{-} \theta_{-} \tilde{\theta}_{+} f_{n}+\tilde{y}_{-} f_{n-1}\right), \\
& A_{7, n}=\operatorname{span}\left(\tilde{\theta}_{-} \theta_{+} f_{n}, i e \tilde{\theta}_{-} \theta_{-} \theta_{+} f_{n}-i \tilde{\theta}_{-} f_{n-1}\right), \\
& A_{8, n}=\operatorname{span}\left(\tilde{\theta}_{-} \theta_{+} \tilde{\theta}_{+} f_{n}, i e \tilde{\theta}_{-} \theta_{-} \theta_{+} \tilde{\theta}_{+} f_{n}-i \tilde{\theta}_{-} \tilde{\theta}_{+} f_{n-1}-\tilde{y} \tilde{\theta}_{-} \theta_{+} f_{n-1}\right) .
\end{aligned}
$$


We then also define for non-negative integer $m$

$$
A_{i, n}^{(m)}:=\tilde{y}^{m} A_{i, n} .
$$

The $\tilde{L}_{ \pm}$action gives $\mathfrak{g l}(1 \mid 1)$-module one-to-one maps, that only change the $L_{N}$-action,

$$
\begin{array}{cl}
\tilde{L}_{+}: \quad A_{3, n}^{(m)} \rightarrow A_{1, n-1}^{(m)}, & \tilde{L}_{-}: A_{4, n}^{(m)} \rightarrow A_{1, n}^{(m)}, \\
\tilde{L}_{+}: A_{5, n}^{(m)} \rightarrow A_{2, n-1}^{(m)}, & \tilde{L}_{-}: A_{6, n}^{(m)} \rightarrow A_{3, n}^{(m)}, \\
\tilde{L}_{+}: A_{6, n}^{(m)} \rightarrow A_{4, n-1}^{(m)}, & \tilde{L}_{-}: A_{7, n}^{(m)} \rightarrow A_{2, n}^{(m)}, \\
\tilde{L}_{+}: A_{8, n}^{(m)} \rightarrow A_{7, n-1}^{(m)}, & \tilde{L}_{-}: A_{8, n}^{(m)} \rightarrow A_{5, n}^{(m)} .
\end{array}
$$

The $\tilde{L}_{N}$-action on $\mathfrak{g l}(1 \mid 1)$-modules is described as follows. $\tilde{L}_{N}$ splits into a semi-simple and a nilpotent part. The semi-simple part acts by multiplication by $-\tilde{n}$, while the nilpotent part acts as follows

$$
\tilde{L}_{N}^{\text {nil }}: A_{i, n}^{(m)} \rightarrow A_{i, n}^{(m-1)} \oplus \begin{cases}0 & \text { if } i=1,2, \\ A_{1, n-1}^{(m)} & \text { if } i=3, \\ A_{1, n}^{(m)} & \text { if } i=4, \\ A_{2, n-1}^{(m)} & \text { if } i=5, \\ A_{3, n}^{(m)} \oplus A_{4, n-1}^{(m)} \oplus A_{1, n-1}^{(m+1)} & \text { if } i=6, \\ A_{1, n-1}^{(m)} \oplus A_{2, n}^{(m)} & \text { if } i=7, \\ A_{5, n}^{(m)} \oplus A_{7, n-1}^{(m)} \oplus A_{3, n-1}^{(m)} \oplus A_{2, n-1}^{(m+1)} \oplus A_{1, n-2}^{(m+1)} & \text { if } i=8 .\end{cases}
$$

By this we mean that the $\mathfrak{g l}(1 \mid 1)$ action on the image of the nilpotent part is the right-hand side. We also understand $A_{i, n}^{(-1)}=0$. An efficient way to picture these computations is the following

Proposition 2.4. Let $\tilde{e}=0$ and $e \neq 0$, then under the left-regular action as $\widetilde{\mathfrak{s l}(1 \mid 1)}$ modules, we have the following two types of 8-dimensional $\widetilde{\mathfrak{s l}(1 \mid 1)}$-modules, $S_{n, a}^{(m)}$

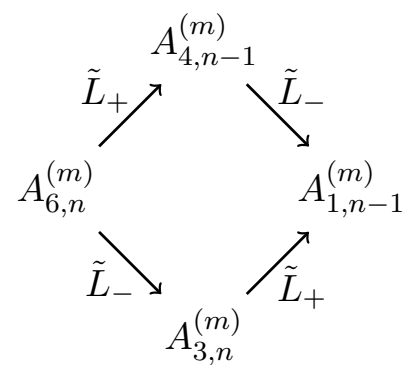

and $S_{n, b}^{(m)}$

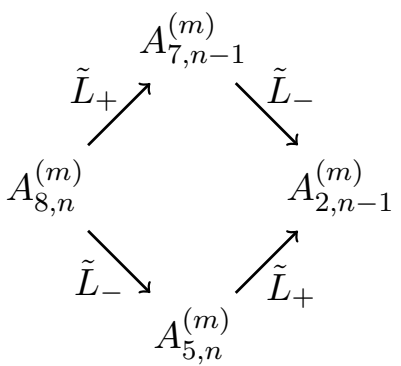


While $L_{N}$-acts semi-simple on these modules, the nilpotent part of $\tilde{L}_{N}$ acts as

$$
\begin{array}{ll}
\tilde{L}_{N}^{\text {nil }}: & S_{n, a}^{(m)} \rightarrow S_{n, a}^{(m-1)} \oplus S_{n, a}^{(m)} \oplus S_{n, a}^{(m+1)}, \\
\tilde{L}_{N}^{\text {nil }}: & S_{n, b}^{(m)} \rightarrow S_{n, b}^{(m-1)} \oplus S_{n, b}^{(m)} \oplus S_{n, b}^{(m+1)} \oplus S_{n, a}^{(m)} \oplus S_{n, a}^{(m+1)} .
\end{array}
$$

However, with a suitable change of basis the situation can be improved. Define

$$
\begin{aligned}
\check{A}_{6, n}^{(m)}:= & \operatorname{span}\left(\tilde{y}^{m}\left(\frac{1}{2} \tilde{y}^{2} f_{n-1}-e f_{n} \tilde{\theta}_{-} \tilde{\theta}_{+}, e \tilde{y} f_{n-1} \tilde{\theta}_{-}+\frac{i}{2} \tilde{y}^{2} f_{n-1} \theta_{-}-i e f_{n} \theta_{-} \tilde{\theta}_{-} \tilde{\theta}_{+}\right)\right), \\
\check{A}_{8, n}^{(m)}:= & \operatorname{span}\left(\tilde { y } ^ { m } \left(i e f_{n} \tilde{\theta}_{-} \theta_{+} \tilde{\theta}_{+}+\tilde{y} f_{n-1} \tilde{\theta}_{+}-\frac{i}{2} \tilde{y}^{2} f_{n-1} \theta_{+}, \frac{1}{2} \tilde{y}^{2} f_{n-2}\right.\right. \\
& \left.\left.+\frac{e}{2} \tilde{y}^{2} f_{n-1} \theta_{-} \theta_{+}+i e \tilde{y} f_{n-1}\left(\tilde{\theta}_{-} \theta_{+}+\theta_{-} \tilde{\theta}_{+}\right)-e f_{n-1} \tilde{\theta}_{-} \tilde{\theta}_{+}-e^{2} f_{n} \theta_{-} \tilde{\theta}_{-} \theta_{+} \tilde{\theta}_{+}\right)\right), \\
\check{A}_{7, n}^{(m)}:= & \operatorname{span}\left(\tilde{y}^{m}\left(e f_{n} \tilde{\theta}_{-} \theta_{+}-i \tilde{y} f_{n-1}, \tilde{y} f_{n-1} \theta_{-}+i f_{n-1} \tilde{\theta}_{-}+i e \theta_{-} \tilde{\theta}_{-} \theta_{+}\right)\right), \\
\check{A}_{i, n}^{(m)}:= & A_{i, n}^{(m)} \quad \text { for } i=1,2,3,4,5 .
\end{aligned}
$$

This redefinition preserves the $\widetilde{\mathfrak{s l}(1 \mid 1)}$ action. However the nilpotent part of $\tilde{L}_{N}$ acts as

$$
\tilde{L}_{N}^{\text {nil }:} \check{A}_{i, n}^{(m)} \rightarrow \check{A}_{i, n}^{(m-1)} \oplus \begin{cases}0 & \text { if } i=1,2, \\ \check{A}_{1, n-1}^{(m)} & \text { if } i=3, \\ \check{A}_{1, n}^{(m)} & \text { if } i=4, \\ \check{A}_{2, n-1}^{(m)} & \text { if } i=5, \\ \check{A}_{3, n}^{(m)} \oplus \check{A}_{1, n-1}^{(m)} & \text { if } i=6, \\ \check{A}_{2, n}^{(m)} & \text { if } i=7, \\ \check{A}_{5, n}^{(m)} \oplus \check{A}_{2, n-1}^{(m)} & \text { if } i=8 .\end{cases}
$$

We thus can considerably improve the previous proposition.

Theorem 2.5. Let $\tilde{e}=0$ and $e \neq 0$, then under the left-regular action of $\widetilde{\mathfrak{g l}(1 \mid 1)}$, we have the following modules, $\check{S}_{n, a}^{(m)}$

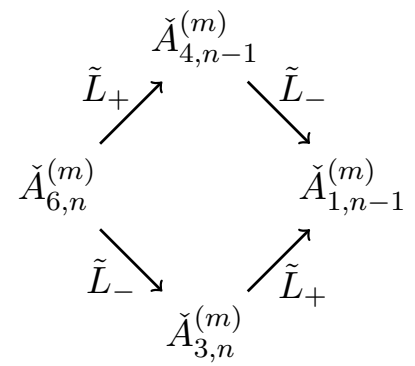

and $\check{S}_{n, b}^{(m)}$

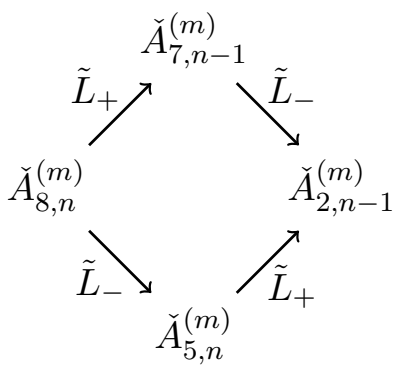


While $L_{N}$-acts semi-simple on these modules, the nilpotent part of $\tilde{L}_{N}$ acts as described in equation (2.3). We can split it into two pieces $\tilde{L}_{N}^{\text {nil }}=\tilde{N}_{a}+\tilde{N}_{b}$, such that

$$
\tilde{N}_{a}: \check{A}_{i, n}^{(m)} \rightarrow \check{A}_{i, n}^{(m-1)}
$$

and

$$
\tilde{N}_{b}: \check{A}_{i, n}^{(m)} \rightarrow \begin{cases}0 & \text { if } i=1,2, \\ \check{A}_{1, n-1}^{(m)} & \text { if } i=3, \\ \check{A}_{1, n}^{(m)} & \text { if } i=4, \\ \check{A}_{2, n-1}^{(m)} & \text { if } i=5, \\ \check{A}_{3, n}^{(m)} \oplus \check{A}_{1, n-1}^{(m)} & \text { if } i=6, \\ \check{A}_{2, n}^{(m)} & \text { if } i=7, \\ \check{A}_{5, n}^{(m)} \oplus \check{A}_{2, n-1}^{(m)} & \text { if } i=8 .\end{cases}
$$

The long-exact sequence describes the action of $\tilde{N}_{a}$,

$$
\cdots \longrightarrow \check{S}_{n, c}^{(m)} \longrightarrow \check{S}_{n, c}^{(m-1)} \longrightarrow \ldots \longrightarrow \check{S}_{n, c}^{(1)} \longrightarrow \check{S}_{n, c}^{(0)} \longrightarrow 0
$$

for $c \in\{a, b\}$.

In order to understand the left-right action, we first have to understand the left-right $\mathfrak{g l}(1 \mid 1)$ action.

Definition 2.6. The highest-weight representation of $\mathfrak{g l}(1 \mid 1)$, with highest-weight $(e, n)$ and lowest-weight $(e, n-1)$ will be denoted by $\rho_{e, n-\frac{1}{2}}$.

We compute: Under the left-right $\mathfrak{g l}(1 \mid 1)$ action the modules decompose as

$$
\begin{aligned}
& X_{2, n}^{(m)}:=\check{A}_{6, n}^{(m)} \oplus \check{A}_{8, n}^{(m)}=\rho_{e,-n+\frac{3}{2}}^{L} \otimes \rho_{-e, n-\frac{3}{2}}^{R}, \\
& X_{-, n}^{(m)}:=\check{A}_{3, n}^{(m)} \oplus \check{A}_{5, n}^{(m)}=\rho_{e,-n+\frac{1}{2}}^{L} \otimes \rho_{-e, n-\frac{3}{2}}^{R}, \\
& X_{+, n-1}^{(m)}:=\check{A}_{4, n-1}^{(m)} \oplus \check{A}_{7, n-1}^{(m)}=\rho_{e,-n+\frac{5}{2}}^{L} \otimes \rho_{-e, n-\frac{3}{2}}^{R}, \\
& X_{0, n-1}^{(m)}:=\check{A}_{1, n-1}^{(m)} \oplus \check{A}_{2, n-1}^{(m)}=\rho_{e,-n+\frac{3}{2}}^{L} \otimes \rho_{-e, n-\frac{3}{2}}^{R},
\end{aligned}
$$

where the last three decompositions follow from the first one and the first one is a straightforward computation. Further, the $\tilde{R}_{ \pm}$action gives $\mathfrak{g l}(1 \mid 1)$-module one-to-one maps, that only change the $R_{N}$-action,

$$
\begin{array}{cc}
\tilde{R}_{-}: \quad A_{4, n}^{(m)} \rightarrow A_{1, n-1}^{(m)}, & \tilde{R}_{+}: A_{3, n}^{(m)} \rightarrow A_{1, n}^{(m)}, \\
\tilde{R}_{-}: A_{6, n}^{(m)} \rightarrow A_{3, n-1}^{(m)}, & \tilde{R}_{+}: A_{5, n}^{(m)} \rightarrow A_{2, n}^{(m)}, \\
\tilde{R}_{-}: A_{7, n}^{(m)} \rightarrow A_{2, n-1}^{(m)}, & \tilde{R}_{+}: A_{6, n}^{(m)} \rightarrow A_{4, n}^{(m)}, \\
\tilde{R}_{-}: \quad A_{8, n}^{(m)} \rightarrow A_{5, n-1}^{(m)}, & \tilde{R}_{+}: A_{8, n}^{(m)} \rightarrow A_{7, n}^{(m)} .
\end{array}
$$

We thus get the picture for left-right modules given in Fig. 1. 


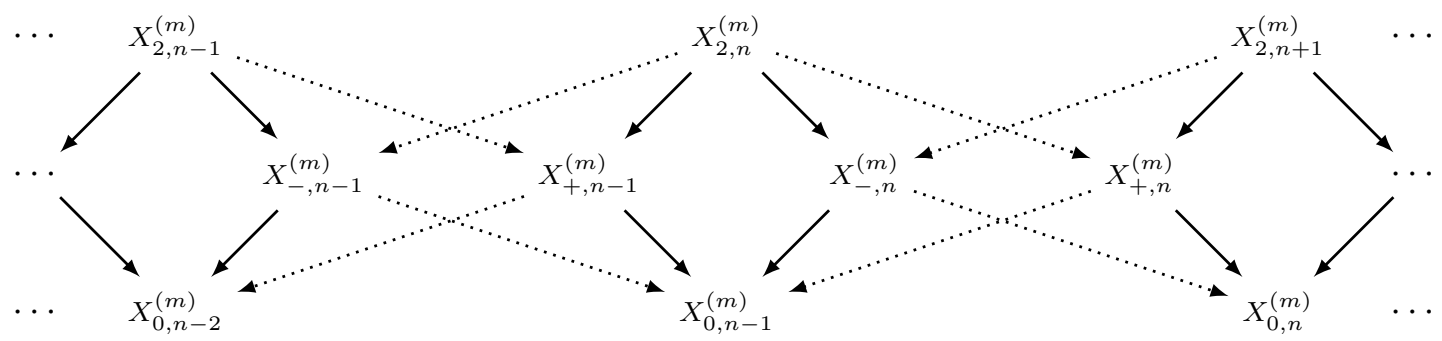

Figure 1. The solid arrows to the right denote the action of $\tilde{L}_{-}$, those to the left of $\tilde{L}_{+}$. While the dotted arrows to the right indicate the action of $\tilde{R}_{+}$and those to the left the one of $\tilde{R}_{-}$.

\subsubsection{Atypical modules}

Let now $\tilde{e}=e=0$. We will start by studying the left-right $\mathfrak{g l}(1 \mid 1)$ action. We define

$$
\begin{array}{ll}
w_{(2,2), n}=\theta_{-} \tilde{\theta}_{-} \theta_{+} \tilde{\theta}_{+} f_{n}, & w_{(2,-), n}=\tilde{\theta}_{-} \theta_{+} \tilde{\theta}_{+} f_{n}, \\
w_{(2,+), n}=\left(\theta_{-} \tilde{\theta}_{-} \tilde{\theta}_{+}-i \tilde{y} \theta_{-} \tilde{\theta}_{-} \theta_{+}\right) f_{n}, & w_{(2,0), n}=\left(\tilde{\theta}_{-} \tilde{\theta}_{+}-i \tilde{y} \tilde{\theta}_{-} \theta_{+}\right) f_{n}, \\
w_{(-, 2), n}=\left(\tilde{\theta}_{-} \theta_{+} \tilde{\theta}_{+}-i \tilde{y} \theta_{-} \theta_{+} \tilde{\theta}_{+}\right) f_{n}, & w_{(-,-), n}=\tilde{y} \theta_{+} \tilde{\theta}_{+} f_{n}, \\
w_{(-,+), n}=\left(\tilde{\theta}_{-} \tilde{\theta}_{+}-i \tilde{y}\left(\theta_{-} \tilde{\theta}_{+}+\tilde{\theta}_{-} \theta_{+}\right)-\tilde{y}^{2} \theta_{-} \theta_{+}\right) f_{n}, & w_{(-, 0), n}=\tilde{y}\left(\tilde{\theta}_{+}-i \tilde{y} \theta_{+}\right) f_{n}, \\
w_{(+, 2), n}=\theta_{-} \tilde{\theta}_{-} \tilde{\theta}_{+} f_{n}, & w_{(+,-), n}=\tilde{\theta}_{-} \tilde{\theta}_{+} f_{n}, \\
w_{(+,+), n}=\tilde{y} \theta_{-} \tilde{\theta}_{-} f_{n}, & w_{(+, 0), n}=\tilde{y} \tilde{\theta}_{-} f_{n}, \\
w_{(0,2), n}=\left(\tilde{\theta}_{-} \tilde{\theta}_{+}-i \tilde{y} \theta_{-} \tilde{\theta}_{+}\right) f_{n}, & w_{(0,-), n}=\tilde{y} \tilde{\theta}_{+} f_{n}, \\
w_{(0,+), n}=\tilde{y}\left(\tilde{\theta}_{-}-i \tilde{y} \theta_{-}\right) f_{n}, & w_{(0,0), n}=\tilde{y}^{2} f_{n} .
\end{array}
$$

Further, define the one-dimensional vector spaces

$$
w_{(a, b), n}^{(m)}=\operatorname{span}\left(\tilde{y} w_{(a, b), n}\right) .
$$

We compute the action of the left- and right-invariant vector fields of the $\mathfrak{g l}(1 \mid 1)$ subalgebra. The result can be best visualized in the following diagram
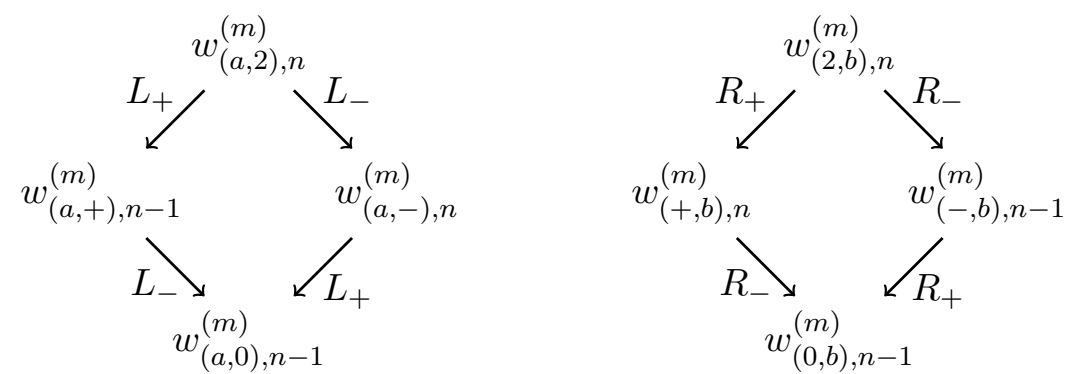

so that we observe

Proposition 2.7. Under the left- and right-invariant vector fields of the $\mathfrak{g l}(1 \mid 1)$ subalgebra the vector space

$$
B_{n}^{(m)}:=\bigoplus_{\substack{a \in\{2,+\} \\ b \in\{2,-\}}} w_{(a, b), n}^{(m)} \oplus \bigoplus_{\substack{a \in\{2,+\} \\ b \in\{+, 0\}}} w_{(a, b), n-1}^{(m)} \oplus \bigoplus_{\substack{a \in\{-, 0\} \\ b \in\{2,-\}}} w_{(a, b), n-1}^{(m)} \oplus \bigoplus_{\substack{a \in\{-, 0\} \\ b \in\{+, 0\}}} w_{(a, b), n-2}^{(m)}
$$

is the tensor product of $\mathfrak{g l}(1 \mid 1)$ projective covers,

$$
B_{n}^{(m)} \cong \mathcal{P}_{2-n}^{L} \otimes \mathcal{P}_{n-2}^{R}
$$




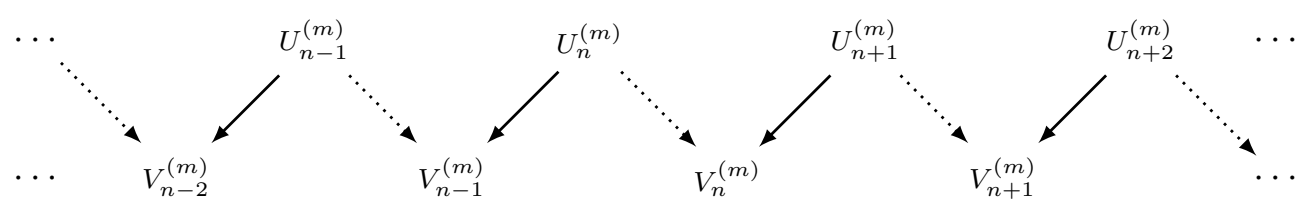

Figure 2. The solid arrows denote the action of $R_{-}$, the dotted ones the one of $L_{-}$.

We define

$$
\mathcal{P}_{n}{ }^{(m)}:=\bigoplus_{a \in \mathbb{Z}} B_{n+a}^{(m)}
$$

The second type of $\mathfrak{g l}(1 \mid 1)$ modules appearing are given by defining the following

$$
\begin{array}{ll}
u_{2, n}^{(m)}:=\operatorname{span}\left(\tilde{y}^{m} \theta_{-} \theta_{+} \tilde{\theta}_{+} f_{n}\right), & u_{R, n}^{(m)}:=\operatorname{span}\left(\tilde{y}^{m} \theta_{-} \tilde{\theta}_{+} f_{n}\right), \\
u_{L, n}^{(m)}:=\operatorname{span}\left(\tilde{y}^{m}\left(\theta_{-} \tilde{\theta}_{+}-i \tilde{y} \theta_{-} \theta_{+}\right) f_{n}\right), & u_{0, n}^{(m)}:=\operatorname{span}\left(\tilde{y}^{m} \tilde{y} \theta_{-} f_{n}\right), \\
v_{2, n}^{(m)}:=\operatorname{span}\left(\tilde{y}^{m} \theta_{+} \tilde{\theta}_{+} f_{n}\right), & v_{R, n}^{(m)}:=\operatorname{span}\left(\tilde{y}^{m} \tilde{\theta}_{+} f_{n}\right), \\
v_{L, n}^{(m)}:=\operatorname{span}\left(\tilde{y}^{m}\left(\tilde{\theta}_{+}-i \tilde{y} \theta_{+}\right) f_{n}\right), & v_{0, n}^{(m)}:=\operatorname{span}\left(\tilde{y}^{m} \tilde{y} f_{n}\right) .
\end{array}
$$

Again, we compute the action of the left- and right-invariant vector fields of the $\mathfrak{g l}(1 \mid 1)$ subalgebra. The result can be best visualized by diagrams. First, we restrict to the action of $R_{+}$ and $L_{+}$, we then get the modules for this subalgebra of $\mathfrak{g l}(1 \mid 1) \oplus \mathfrak{g l}(1 \mid 1)$
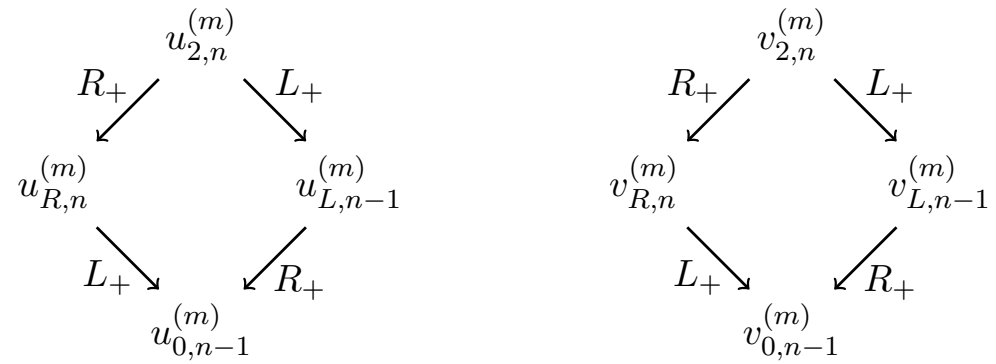

We call the first one $U_{n}^{(m)}$ and the second one $V_{n}^{(m)}$. The vector fields $L_{-}$and $R_{-}$act trivially on the second one while the first one gives one-to-one maps illustrated in Fig. 2. Thus the set

$$
\mathcal{P}_{n}^{-(m)}:=\bigoplus_{a \in \mathbb{Z}} U_{n+a}^{(m)} \oplus \bigoplus_{b \in \mathbb{Z}} V_{n+b}^{(m)}
$$

forms an infinite-dimensional $\mathfrak{g l}(1 \mid 1)$ left-right module called $\mathcal{P}_{n}^{-(m)}$.

The third type of $\mathfrak{g l}(1 \mid 1)$ modules appearing is very similar to the previous one and are given by defining the following

$$
\begin{array}{ll}
s_{2, n}^{(m)}:=\operatorname{span}\left(\tilde{y}^{m} \theta_{-} \tilde{\theta}_{-} \theta_{+} f_{n}\right), & s_{L, n}^{(m)}:=\operatorname{span}\left(\tilde{y}^{m} \tilde{\theta}_{-} \theta_{+} f_{n}\right), \\
s_{R, n}^{(m)}:=\operatorname{span}\left(\tilde{y}^{m}\left(\tilde{\theta}_{-} \theta_{+}-i \tilde{y} \theta_{-} \theta_{+}\right) f_{n}\right), & s_{0, n}^{(m)}:=\operatorname{span}\left(\tilde{y}^{m} \tilde{y} \theta_{+} f_{n}\right), \\
t_{2, n}^{(m)}:=\operatorname{span}\left(\tilde{y}^{m} \theta_{-} \tilde{\theta}_{-} f_{n}\right), & t_{L, n}^{(m)}:=\operatorname{span}\left(\tilde{y}^{m} \tilde{\theta}_{-} f_{n}\right), \\
t_{R, n}^{(m)}:=\operatorname{span}\left(\tilde{y}^{m}\left(\tilde{\theta}_{-}-i \tilde{y} \theta_{-}\right) f_{n}\right), & t_{0, n}^{(m)}:=\operatorname{span}\left(\tilde{y}^{m} \tilde{y} f_{n}\right) .
\end{array}
$$




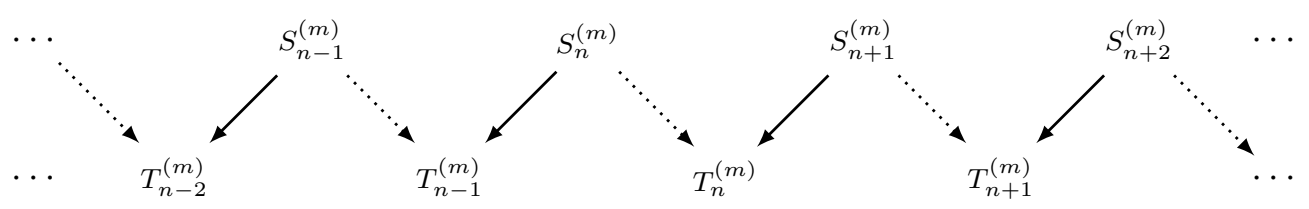

Figure 3. The solid arrows denote the action of $L_{+}$, the dotted ones the one of $R_{+}$.

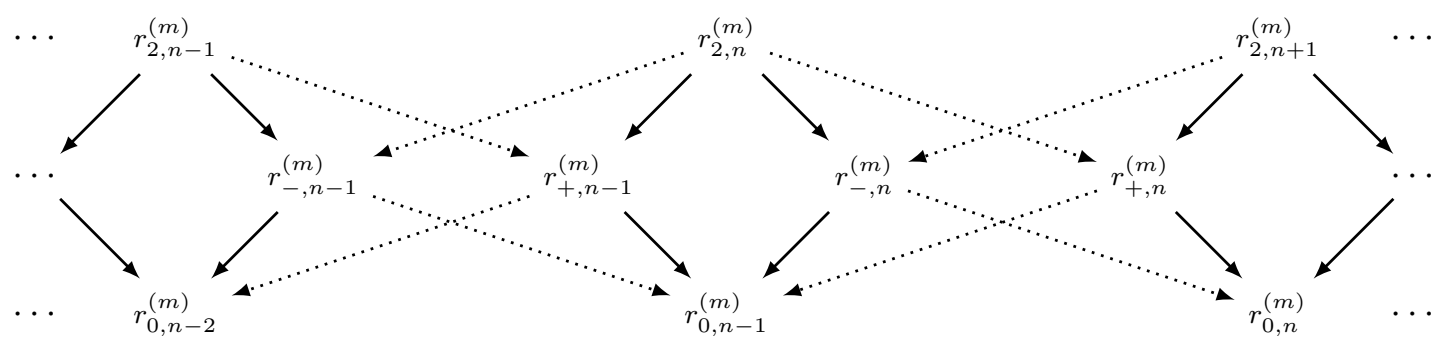

Figure 4. The solid arrows to the right denote the action of $L_{-}$, those to the left of $L_{+}$. While the dotted arrows to the right indicate the action of $R_{+}$and those to the left the one of $R_{-}$.

Again, we compute the action of the left- and right-invariant vector fields of the $\mathfrak{g l}(1 \mid 1)$ subalgebra. The result can also again be best visualized by diagrams. First, we restric to the action of $R_{-}$and $L_{-}$, we then get the modules for this subalgebra of $\mathfrak{g l}(1 \mid 1) \oplus \mathfrak{g l}(1 \mid 1)$
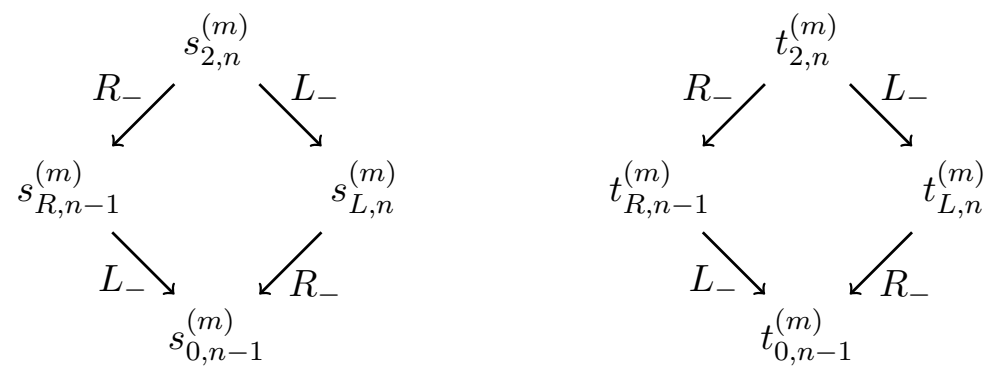

We call the first one $S_{n}^{(m)}$ and the second one $T_{n}^{(m)}$. The vector fields $L_{+}$and $R_{+}$act trivially on the second one while the first one gives one-to-one maps illustrated in Fig. 3 . Thus the set

$$
\mathcal{P}_{n}^{+(m)}:=\bigoplus_{a \in \mathbb{Z}} S_{n+a}^{(m)} \oplus \bigoplus_{b \in \mathbb{Z}} T_{n+b}^{(m)}
$$

forms an infinite-dimensional $\mathfrak{g l}(1 \mid 1)$ left-right module called $\mathcal{P}_{n}^{+(m)}$.

The forth type of $\mathfrak{g l}(1 \mid 1)$ module is given by

$$
\begin{aligned}
r_{2, n}^{(m)}:=\operatorname{span}\left(\tilde{y}^{m} \theta_{-} \theta_{+} f_{n}\right), & r_{-, n}^{(m)}:=\operatorname{span}\left(\tilde{y}^{m} \theta_{+} f_{n}\right), \\
r_{+, n}^{(m)}:=\operatorname{span}\left(\tilde{y}^{m} \theta_{-} f_{n}\right), & r_{0, n}^{(m)}:=\operatorname{span}\left(\tilde{y}^{m} f_{n}\right) .
\end{aligned}
$$

Then as a left-right module we get the picture as in Fig. 4 . We denote this module by $\mathcal{P}_{n}^{0^{(m)}}$. Observe that we have the inclusion of $\mathfrak{g l}(1 \mid 1)$ left-right modules

$$
\mathcal{P}_{n}^{0^{(m)}} \subset \mathcal{P}_{n}^{+{ }^{(m-1)}} \oplus \mathcal{P}_{n}^{-(m-1)}, \quad \mathcal{P}_{n}^{ \pm(m)} \subset \mathcal{P}_{n}^{(m-1)}
$$


for $m>0$. Especially as a $\mathfrak{g l}(1 \mid 1)$ left-right module the atypical part, that is the $e=\tilde{e}=0$ part of the space of functions on $\widehat{\mathfrak{g l}(1 \mid 1)}$ decomposes as

$$
\int_{0}^{1}\left(\mathcal{P}_{n}^{0(0)} \oplus \mathcal{P}_{n}^{1(0)} \oplus \bigoplus_{m=1}^{\infty} \mathcal{P}_{n}{ }^{(m)}\right) d n .
$$

Here $\mathcal{P}_{n}^{1(0)}$ denotes the module generated by $\mathcal{P}_{n}^{+(0)}$ and $\mathcal{P}_{n}^{-(0)}$ whose intersection is the submodule $\mathcal{P}^{0(1)}$. These modules are constructed such that the tilded action is easy to read of, the $\tilde{L}_{ \pm}$ and $\tilde{R}_{ \pm}$are $\mathfrak{s l}(1 \mid 1)$ module homomorphism (they only change the action of $L_{N}$ or $R_{N}$ ) and for $m>0$ they act as

$$
\begin{aligned}
& \operatorname{Image}\left(\tilde{L}_{ \pm}: \mathcal{P}_{n}{ }^{(m)} \longrightarrow \mathcal{P}_{n}{ }^{(m-1)}\right)=\operatorname{Image}\left(\tilde{R}_{ \pm}: \mathcal{P}_{n}{ }^{(m)} \longrightarrow \mathcal{P}_{n}{ }^{(m-1)}\right)=\mathcal{P}_{n}^{ \pm}{ }^{(m)} \\
& \operatorname{Image}\left(\tilde{L}_{ \pm}: \mathcal{P}_{n}^{\mp}{ }^{(m)} \longrightarrow \mathcal{P}_{n}^{\mp}{ }^{(m-1)}\right)=\operatorname{Image}\left(\tilde{R}_{ \pm}: \mathcal{P}_{n}^{\mp}{ }^{(m)} \longrightarrow \mathcal{P}_{n}^{\mp(m-1)}\right)=\mathcal{P}_{n}^{0^{(m)}}
\end{aligned}
$$

while in the $m=0$ case we have

$$
\begin{aligned}
& \operatorname{Image}\left(\tilde{L}_{ \pm}: \mathcal{P}_{n}{ }^{(0)} \longrightarrow \mathcal{P}_{n}^{ \pm}{ }^{(0)}\right)=\operatorname{Image}\left(\tilde{R}_{ \pm}: \mathcal{P}_{n}{ }^{(0)} \longrightarrow \mathcal{P}_{n}^{ \pm}{ }^{(0)}\right)=\mathcal{P}_{n}^{ \pm}{ }^{(0)} \\
& \operatorname{Image}\left(\tilde{L}_{ \pm}: \mathcal{P}_{n}^{\mp}{ }^{(0)} \longrightarrow \mathcal{P}_{n}^{0^{(0)}}\right)=\operatorname{Image}\left(\tilde{R}_{ \pm}: \mathcal{P}_{n}^{\mp}{ }^{(0)} \longrightarrow \mathcal{P}_{n}{ }^{(0)}\right)=\mathcal{P}_{n}^{0^{(0)}}
\end{aligned}
$$

The image of these maps is in the kernel and we inspect that it is precisely the kernel, hence both $\tilde{L}_{+}$and $\tilde{R}_{+}$action is described by the long-exact sequences

$$
\begin{aligned}
& \cdots \longrightarrow \mathcal{P}_{n}{ }^{(m)} \longrightarrow \mathcal{P}_{n}{ }^{(m-1)} \longrightarrow \ldots \longrightarrow \mathcal{P}_{n}{ }^{(1)} \longrightarrow \mathcal{P}_{n}{ }^{(0)} \longrightarrow \mathcal{P}_{n}^{+(0)} \longrightarrow 0 \\
& \cdots \longrightarrow \mathcal{P}_{n}^{-(m)} \longrightarrow \mathcal{P}_{n}^{-(m-1)} \longrightarrow \ldots \longrightarrow \mathcal{P}_{n}^{-(1)} \longrightarrow \mathcal{P}_{n}^{-(0)} \longrightarrow \mathcal{P}_{n}^{0^{(0)}} \longrightarrow 0
\end{aligned}
$$

while the one of $\tilde{L}_{-}$and $\tilde{R}_{-}$action is described by the long-exact sequences

$$
\begin{aligned}
& \cdots \longrightarrow \mathcal{P}_{n}{ }^{(m)} \longrightarrow \mathcal{P}_{n}{ }^{(m-1)} \longrightarrow \ldots \longrightarrow \mathcal{P}_{n}{ }^{(1)} \longrightarrow \mathcal{P}_{n}{ }^{(0)} \longrightarrow \mathcal{P}_{n}^{-(0)} \longrightarrow 0 \\
& \cdots \longrightarrow \mathcal{P}_{n}^{+(m)} \longrightarrow \mathcal{P}_{n}^{+(m-1)} \longrightarrow \ldots \longrightarrow \mathcal{P}_{n}^{+(1)} \longrightarrow \mathcal{P}_{n}^{+(0)} \longrightarrow \mathcal{P}_{n}^{0^{(0)}} \longrightarrow 0 .
\end{aligned}
$$

The action of $\tilde{L}_{N}$ and $\tilde{R}_{N}$ is uniquely specified by its action on the top components, the map can be split into two components $\tilde{L}_{N}=\tilde{L}_{N}^{a}+\tilde{L}_{N}^{b}$ and $\tilde{R}_{N}=\tilde{R}_{N}^{a}+\tilde{R}_{N}^{b}$ such that $\tilde{L}_{N}^{a}$ and $\tilde{R}_{N}^{a}$ are both one-to-one maps from $x_{i}^{(m)}$ to $x_{i}^{(m-1)}$ for all $x$ in $\{s, t, u, v, w\}$ and $i$ labelling the corresponding indices. More precisely they act as multiplication by $\pm i m\left(+\right.$ for $\tilde{R}$ and - for $\tilde{L}$ ) times $\tilde{y}^{-1}$. Especially they act trivially when $m=0$. The images of the second part are (for $m \neq 0$ )

$$
\begin{aligned}
& \operatorname{Image}\left(\tilde{L}_{N}^{b}: \mathcal{P}_{n}{ }^{(m)} \longrightarrow \mathcal{P}_{n}{ }^{(m-1)}\right)=\operatorname{Image}\left(\tilde{R}_{N}^{b}: \mathcal{P}_{n}{ }^{(m)} \longrightarrow \mathcal{P}_{n}{ }^{(m-1)}\right)=\mathcal{P}_{n}^{1^{(m)}}, \\
& \operatorname{Image}\left(\tilde{L}_{N}^{b}: \mathcal{P}_{n}^{ \pm}{ }^{(m)} \longrightarrow \mathcal{P}_{n}^{ \pm}{ }^{(m-1)}\right)=\operatorname{Image}\left(\tilde{R}_{N}^{b}: \mathcal{P}_{n}^{ \pm}(m) \longrightarrow \mathcal{P}_{n}^{ \pm}{ }^{(m-1)}\right)=\mathcal{P}_{n}^{0^{(m)}}, \\
& \operatorname{Image}\left(\tilde{L}_{N}^{b}: \mathcal{P}_{n}^{0^{(m)}} \longrightarrow \mathcal{P}_{n}^{0^{(m-1)}}\right)=\operatorname{Image}\left(\tilde{R}_{N}^{b}: \mathcal{P}_{n}^{0^{(m)}} \longrightarrow \mathcal{P}_{n}^{0^{(m-1)}}\right)=0,
\end{aligned}
$$

and for $m=0$

$$
\begin{aligned}
& \operatorname{Image}\left(\tilde{L}_{N}^{b}: \mathcal{P}_{n}{ }^{(0)} \longrightarrow \mathcal{P}_{n}^{1^{(0)}}\right)=\operatorname{Image}\left(\tilde{R}_{N}^{b}: \mathcal{P}_{n}^{(0)} \longrightarrow \mathcal{P}_{n}^{1^{(0)}}\right)=\mathcal{P}_{n}^{1^{(0)}}, \\
& \operatorname{Image}\left(\tilde{L}_{N}^{b}: \mathcal{P}_{n}^{ \pm}(0) \longrightarrow \mathcal{P}_{n}^{0(0)}\right)=\operatorname{Image}\left(\tilde{R}_{N}^{b}: \mathcal{P}_{n}^{ \pm}(0) \longrightarrow \mathcal{P}_{n}^{0(0)}\right)=\mathcal{P}_{n}^{0^{(0)}}, \\
& \operatorname{Image}\left(\tilde{L}_{N}^{b}: \mathcal{P}_{n}^{0^{(0)}} \longrightarrow \mathcal{P}_{n}^{0^{(0)}}\right)=\operatorname{Image}\left(\tilde{R}_{N}^{b}: \mathcal{P}_{n}^{0^{(0)}} \longrightarrow \mathcal{P}_{n}^{0(0)}\right)=0 .
\end{aligned}
$$




\section{Free field realization}

In this section we construct free field realization of the holomorphic and anti-holomorphic currents of affine Takiff super Lie algebra $\hat{\tilde{g} l}(1 \mid 1)$, and its modules. Such free field realizations are constructed by starting with a triangular decomposition of a Lie supergroup element as in (2.1). This procedure is outlined in [39], and we can immediately infer the bulk screening charge from (3.2.32) of [8], as well as the currents in the free field realization from (3.2.39) of that work. We however still need to prove that the screening charge has the desired properties. The harmonic analysis is expected to be a semi-classical (large level) limit of the screened free field theory. Examples on this for type one Lie supergroups are [12, 39, 44, 45].

The Takiff algebra admits an automorphism $\omega_{\alpha}$ for $\alpha$ in $\mathbb{C}$. It is given by

$$
E \mapsto E+\alpha \tilde{E}, \quad \psi^{+} \mapsto \psi^{+}+\alpha \tilde{\psi}^{+}
$$

and leaving all others invariant. It allows us to restrict to the bilinear form $\tilde{\kappa}$. In order to see this, consider the bilinear form $\kappa_{\beta}:=\tilde{\kappa}+\beta \kappa_{0}$, then

$$
\kappa\left(\omega_{-\beta}(X), \omega_{-\beta}(Y)\right)=\tilde{\kappa}(X, Y)
$$

for all $X, Y$ in $\widetilde{\mathfrak{g l}(1 \mid 1)}$. In [4], the affinization of $\widetilde{\mathfrak{g l}(1 \mid 1)}$ was discussed and levels $\tilde{k}$ and $k$ associated to the bilinear forms $\tilde{\kappa}$ and $\kappa$ were introduced. The above automorphism relates the affine vertex algebra of $\widetilde{\mathfrak{g l}(1 \mid 1)}$ at levels $(\tilde{k}, k)$ to the one at levels $(\tilde{k}, 0)$.

\subsection{Currents and screening operator}

Consider a holomorphic $b c$-ghost vertex algebra taking values in the odd part of the Takiff superalgebra. Its components have OPE

$$
b(z) \tilde{c}(w) \sim \tilde{b}(z) c(w) \sim \frac{1}{(z-w)}
$$

and all other OPEs are regular. We also need free bosons (generators of the Heisenberg VOA) taking values in the even part of the Takiff superalgebra. Its components have OPE in their holomorphic part

$$
\partial X(z) \partial \tilde{Y}(w) \sim \partial \tilde{X}(z) \partial Y(w) \sim \frac{-1 / k}{(z-w)^{2}} .
$$

In the same way we define anti holomorphic partners of the ghosts. The fields $X(z, \bar{z}), Y(z, \bar{z})$, $\tilde{X}(z, \bar{z}), \tilde{Y}(z, \bar{z})$ will be considered as both holomorphic and anti holomorphic, i.e., depending on $z$ and $\bar{z}$, with the same OPEs in anti-holomorphic sector.

Currents should be thought of as quantizations of invariant vector fields. We define holomorphic currents by the formulas

$$
\begin{array}{llrl}
J^{N} & =-i \partial \tilde{X}+b \tilde{c}+\tilde{b} c+i \partial Y, & & J^{E}=-i k \partial \tilde{Y}, \\
J^{+} & =\tilde{b}, & & J^{-}=k \partial \tilde{c}-i k \tilde{c} \partial Y-i k c \partial \tilde{Y}, \\
\tilde{J}^{N}=-i \partial X+b c, & & \tilde{J}^{E}=-i k \partial Y, \\
\tilde{J}^{+}=b, & & \tilde{J}^{-}=k \partial c-i k c \partial Y .
\end{array}
$$

and the anti holomorphic ones by

$$
\bar{J}^{N}=i k \bar{\partial} \tilde{X}-i \bar{\partial} Y-\bar{b} \overline{\tilde{c}}-\overline{\tilde{b}} \bar{c}, \quad \bar{J}^{E}=i k \bar{\partial} \tilde{Y}
$$




$$
\begin{array}{rlrl}
\bar{J}^{+} & =k \bar{\partial} \tilde{\bar{c}}-i k \tilde{\bar{c}} \bar{\partial} Y-i k \bar{c} \bar{\partial} \tilde{Y}, & \bar{J}^{-} & =-\overline{\tilde{b}}, \\
\tilde{\bar{J}}^{N} & =i k \bar{\partial} X-\bar{b} \bar{c}, & \tilde{\bar{J}}^{E}=i k \bar{\partial} Y, \\
\tilde{\bar{J}}^{+}=k \bar{\partial} \bar{c}-i k \bar{c} \bar{\partial} Y, & \tilde{\bar{J}}^{-}=-\bar{b} .
\end{array}
$$

By direct calculation one can see that

Proposition 3.1. The OPEs of currents (3.1) are those of the level $k$ with respect to $\tilde{\kappa}$ affine Takiff superalgebra, that is the only non-regular OPEs are

$$
\begin{aligned}
J^{N}(z) J^{ \pm}(w) & \sim \pm \frac{J^{ \pm}(w)}{(z-w)}, & J^{+}(z) J^{-}(w) & \sim \frac{J^{E}(w)}{(z-w)}, \\
J^{N}(z) \tilde{J}^{E}(w) & \sim \frac{k}{(z-w)^{2}}, & J^{N}(z) \tilde{J}^{ \pm}(w) & \sim \pm \frac{\tilde{J}^{ \pm}(w)}{(z-w)}, \\
\tilde{J}^{N}(z) J^{E}(w) & \sim \frac{k}{(z-w)^{2}}, & \tilde{J}^{N}(z) J^{ \pm}(w) & \sim \pm \frac{\tilde{J}^{ \pm}(w)}{(z-w)}, \\
J^{+}(z) \tilde{J}^{-}(w) & \sim \frac{k}{(z-w)^{2}}+\frac{\tilde{J}^{E}(w)}{(z-w)}, & J^{-}(z) \tilde{J}^{+}(w) & \sim-\frac{k}{(z-w)^{2}}+\frac{\tilde{J}^{E}(w)}{(z-w)} .
\end{aligned}
$$

and the same set of OPEs of anti-holomorphic currents (3.2).

One can substitute these currents into the energy-momentum tensor obtained in [4] (see also [36])

$$
T(z)=\frac{1}{k}:\left(J^{N} \tilde{J}^{E}+J^{E} \tilde{J}^{N}-J^{+} \tilde{J}^{-}+J^{-} \tilde{J}^{+}+\frac{1}{k} \tilde{J}^{E} \tilde{J}^{E}\right):
$$

getting

$$
\left.T(z)=:\left(\partial \tilde{X} \partial Y+\partial \tilde{Y} \partial X-k \partial^{2} Y-\tilde{b} \partial c-b \partial \tilde{c}\right)\right):(z),
$$

which clearly has central charge zero. The key role in free field realization is played by screening operators.

Theorem 3.2. The currents (3.1) lie in the joint kernel of the intertwiners

$$
S=\oint b(z) e^{i Y(z)} d z, \quad \tilde{S}=\oint(i \tilde{Y}(z) b(z)+\tilde{b}(z)) e^{i Y(z)} d z .
$$

Proof. The computations are straightforward. We demonstrate here only action of $S$ and $\tilde{S}$ on $J^{-}$and $\tilde{J}^{-}$. For $S$ we get

$$
\begin{aligned}
S J^{-}(w) & =\oint k e^{i Y(z)}\left(\frac{1}{(z-w)^{2}}+\frac{-i \partial Y(w)}{(z-w)}+\text { regular }\right) d z \\
& =\oint\left(e^{i Y(w)} \frac{k}{(z-w)^{2}}+\text { regular }\right) d z=0, \\
S \tilde{J}^{-}(w) & =\oint \text { regular } d z=0,
\end{aligned}
$$

and for the action of $\tilde{S}$ it becomes

$$
\tilde{S} J^{-}(w)=\oint e^{i Y(z)}\left(\frac{i k \tilde{Y}(z)}{(z-w)^{2}}-\frac{i k \partial \tilde{Y}(w)-k \tilde{Y}(w) \partial Y(w)}{(z-w)}+\text { regular }\right) d z
$$




$$
\begin{aligned}
& =\oint\left(e^{i Y(w)} \frac{i k \tilde{Y}(w)}{(z-w)^{2}}+\text { regular }\right) d z=0, \\
\tilde{S} \tilde{J}^{-}(w) & =\oint e^{i Y(z)}\left(\frac{k}{(z-w)^{2}}-\frac{i k \partial Y(w)}{(z-w)}+\text { regular }\right) d z \\
& =\oint\left(e^{i Y(w)} \frac{k}{(z-w)^{2}}+\text { regular }\right) d z=0 .
\end{aligned}
$$

The same theorem can be proved for the anti-holomorphic part. We can now define bulk screening charge which involves both holomorphic and anti-holomorphic parts:

$$
Q=\int(\bar{b}(\bar{z}) \tilde{b}(z)+\tilde{\bar{b}}(\bar{z}) b(z)+i \bar{b}(\bar{z}) b(z) \tilde{Y}(z, \bar{z})) e^{i Y(z, \bar{z})} d^{2} z .
$$

This $Q$ should define correlation functions, which will not be considered in this paper.

\section{$3.2 \quad$ Vertex operators}

We define the vertex operator

$$
V_{e, n, \tilde{e}, \tilde{n}}=: e^{i e X-i n Y+i \tilde{e} \tilde{X}-i \tilde{n} \tilde{Y}}:,
$$

whose conformal dimension can be calculated with (3.3):

$$
\Delta=e \tilde{n}+n \tilde{e}+i k \tilde{e} .
$$

It transforms as a primary field corresponding to a highest-weight state, that is

$$
\begin{aligned}
& J^{E}(z) V_{e, n, \tilde{e}, \tilde{n}}(w) \sim \frac{k e V_{e, n, \tilde{e}, \tilde{n}}(w)}{(z-w)}, \\
& J^{N}(z) V_{e, n, \tilde{e}, \tilde{n}}(w) \sim \frac{(n-\tilde{e}) V_{e, n, \tilde{e}, \tilde{n}(w)}}{(z-w)}, \\
& \tilde{J}^{E}(z) V_{e, n, \tilde{e}, \tilde{n}}(w) \sim \frac{k \tilde{e} V_{e, n, \tilde{e}, \tilde{n}}(w)}{(z-w)}, \\
& \tilde{J}^{N}(z) V_{e, n, \tilde{e}, \tilde{n}}(w) \sim \frac{\tilde{n} V_{e, n, \tilde{e}, \tilde{n}}(w)}{(z-w)} \\
& J^{-}(z) V_{e, n, \tilde{e}, \tilde{n}}(w) \sim \frac{k e: c(w) V_{e, n, \tilde{e}, \tilde{n}}(w):+\tilde{e} k: \tilde{c}(w) V_{e, n, \tilde{e}, \tilde{n}}(w):}{(z-w)}, \\
& \tilde{J}^{-}(z) V_{e, n, \tilde{e}, \tilde{n}}(w) \sim \frac{k \tilde{e}: c(w) V_{e, n, \tilde{e}, \tilde{n}}(w):}{(z-w)}, \\
& J^{-}(z): c(w) V_{e, n, \tilde{e}, \tilde{n}}(w): \sim \frac{k \tilde{e}: \tilde{c}(w) c(w) V_{e, n, \tilde{e}, \tilde{n}}(w):}{(z-w)} .
\end{aligned}
$$

\subsection{Free field realization of typical modules}

We can now combine holomorphic and anti-holomorphic ghost fields with the vertex operator introduced above to form bulk free field realization for typical modules. It is convenient to represent it in the matrix form

$$
\phi_{e, n, \tilde{e}, \tilde{n}}(z)=: \tilde{Y}^{m} V_{e, n, \tilde{e}, \tilde{n}}:(z, \bar{z})\left(\begin{array}{cccc}
1 & \bar{c} & \tilde{\bar{c}} & \tilde{\bar{c}} \bar{c} \\
c & c \bar{c} & c \tilde{\bar{c}} & c \overline{\bar{c}} \bar{c} \\
\tilde{c} & \tilde{c} \bar{c} & \tilde{c} \overline{\bar{c}} & \tilde{c} \overline{\bar{c}} \bar{c} \\
c \tilde{c} & c \tilde{c} \bar{c} & c \tilde{c} \tilde{\bar{c}} & c \tilde{\bar{c}} \overline{\bar{c}} \bar{c}
\end{array}\right):
$$


Here each row and column of the matrix $C$ is understood as a span of its elements. Using the OPEs one can easily check that columns carry typical representations for the holomorphic currents and the rows - for the anti-holomorphic ones. The elements $C_{1, i}$ are the highest weights for the columns, and the elements $C_{i, 1}$ - for the rows. For $m=0$ they are Virasoro primary fields with conformal dimension (3.4).

\subsection{A free field realization for atypical and semitypical modules}

Consider two pairs of symplectic fermions $\chi_{ \pm}, \tilde{\chi}_{ \pm}$with OPE

$$
\chi_{+}(z) \tilde{\chi}_{-}(w) \sim \chi_{-}(z) \tilde{\chi}_{+}(w) \sim \frac{k}{(z-w)^{2}}
$$

and a pair of pairs of free bosons $\partial X, \partial Y, \partial \tilde{X}, \partial \tilde{Y}$ with OPE

$$
\partial X(z) \partial \tilde{Y}(w) \sim \partial Y(z) \partial \tilde{X}(w) \sim \frac{1}{(z-w)^{2}} .
$$

Recall, that symplectic fermions are a logarithmic conformal field theory. Especially, the vacuum is part of a larger indecomposable module whose four composition factors are all isomorphic to the vacuum itself, for details see [31]. The generating fields of these modules are $\Omega_{ \pm}(z, \bar{z})$, $\tilde{\theta}_{ \pm}(z, \bar{z}), \theta_{ \pm}(z, \bar{z})$ with the following behaviour under the action of the symplectic fermions:

$$
\begin{array}{ll}
\chi_{ \pm}(z) \Omega_{ \pm}(w, \bar{w}) \sim \frac{k \theta_{ \pm}(w, \bar{w})}{(z-w)}, & \tilde{\chi}_{\mp}(z) \Omega_{ \pm}(w, \bar{w}) \sim \frac{k \tilde{\theta}_{\mp}(w, \bar{w})}{(z-w)}, \\
\chi_{ \pm}(z) \tilde{\theta}_{\mp}(w, \bar{w}) \sim \frac{k}{(z-w)}, & \tilde{\chi}_{\mp}(z) \theta_{ \pm}(w, \bar{w}) \sim-\frac{k}{(z-w)} .
\end{array}
$$

We split the field $\tilde{Y}(z, \bar{z})$ into chiral and anti-chiral part, that is $\tilde{Y}(z, \bar{z})=\tilde{Y}(z)+\tilde{\bar{Y}}(\bar{z})$. Then we define

$$
\begin{aligned}
& K^{E}(z)=k \partial \tilde{Y}(z), \quad K^{N}(z)=\partial \tilde{X}(z), \quad \tilde{K}^{E}(z)=k \partial Y(z), \quad \tilde{K}^{N}(z)=\partial X(z), \\
& K^{ \pm}(z)=e^{ \pm Y(z)}\left(\chi_{ \pm}(z)-\tilde{Y}(z) \tilde{\chi}_{ \pm}(z)\right), \quad \tilde{K}^{ \pm}(z)=\mp e^{ \pm Y(z)} \tilde{\chi}_{ \pm}(z) .
\end{aligned}
$$

and in analogy also anti-holomorphic currents. We compute

Proposition 3.3. The OPEs of currents (3.5) are those of the level $k$ with respect to $\tilde{\kappa}$ affine Takiff superalgebra. These OPEs are listed in Proposition 3.1. The energy-momentum tensor for these currents is

$$
T(z)=: \partial \tilde{X}(z) \partial Y(z):+: \partial \tilde{Y}(z) \partial X(z):-\frac{1}{k}\left(: \chi_{+}(z) \tilde{\chi}_{-}(z):+: \chi_{-}(z) \tilde{\chi}_{+}(z):\right) .
$$

These currents are in the kernel of

$$
Q:=\oint\left(: \tilde{\chi}_{+}(z) \tilde{\theta}_{+}(z, \bar{z}):+: \tilde{\chi}_{-}(z) \tilde{\theta}_{-}(z, \bar{z}):+2 k \partial X(z)\right) d z
$$

acting on the chiral algebra generated under operator product by $e^{ \pm Y(z)}, \tilde{Y}(z), \chi_{ \pm}(z), \tilde{\chi}_{ \pm}(z)$, $\partial X(z), \partial \tilde{X}(z)$ and their derivatives. The currents are also invariant under the $U(1)$-action induced by $\varphi$ which is defined by

$$
\varphi\left(e^{ \pm Y(z)}\right)=\mp e^{ \pm Y(z)}, \quad \varphi\left(\chi_{ \pm}(z)\right)= \pm \chi_{ \pm}(z), \quad \varphi\left(\tilde{\chi}_{ \pm}(z)\right)= \pm \tilde{\chi}_{ \pm}(z) .
$$


As discussed in [4], semi-typical and atypical modules appear when $\tilde{e} / k=m \in \mathbb{Z}$. The vertex operator

$$
V_{e, n, \tilde{e}, \tilde{n}}(z):=: e^{\frac{e}{k} X(z)+n Y(z)+\frac{\tilde{e}}{k} \tilde{X}(z)+\tilde{n} \tilde{Y}(z)}:
$$

has weight $(e, n, \tilde{e}, \tilde{n})$ under the currents $K^{E}, K^{N}, \tilde{K}^{E}, \tilde{K}^{N}$. Define

$$
\phi_{m}^{ \pm}(z):=: \chi_{ \pm}(z) \partial \chi_{ \pm}(z) \cdots \partial^{m} \chi_{ \pm}(z):, \quad \tilde{\phi}_{m}^{ \pm}(z):=: \tilde{\chi}_{ \pm}(z) \partial \tilde{\chi}_{ \pm}(z) \cdots \partial^{m} \tilde{\chi}_{ \pm}(z): .
$$

Then it is a computation to verify that for $m \leq 0$

$$
\varphi_{e, n, m k, \tilde{n}}(z):=V_{e, n, m k, \tilde{n}}(z) \phi_{-m}^{+}(z) \tilde{\phi}_{-m}^{+}
$$

is a primary field for the highest-weight representation of weight $(e, n, m k, \tilde{n})$ of the correct conformal dimension $e \tilde{n} / k+m n+m(m-1)$. It is a submodule of the modules generated by

$$
\varphi_{e, n, m k, \tilde{n}}(z) \theta_{+}(z, \bar{z}), \quad \varphi_{e, n, m k, \tilde{n}}(z) \tilde{\theta}_{+}(z, \bar{z}), \quad \varphi_{e, n, m k, \tilde{n}}(z) \theta_{+}(z, \bar{z}) \tilde{\theta}_{+}(z, \bar{z}) .
$$

If $m \geq 0$, then we can construct analogous primary fields for lowest-weight representations by replacing \pm , i.e., the primary field is

$$
\varphi_{e, n, m k, \tilde{n}}(z):=V_{e, n, m k, \tilde{n}}(z) \phi_{m}^{-}(z) \tilde{\phi}_{m}^{-}
$$

and it is a submodule of the modules generated by

$$
\varphi_{e, n, m k, \tilde{n}}(z) \theta_{-}(z, \bar{z}), \quad \varphi_{e, n, m k, \tilde{n}}(z) \tilde{\theta}_{-}(z, \bar{z}), \quad \varphi_{e, n, m k, \tilde{n}}(z) \theta_{-}(z, \bar{z}) \tilde{\theta}_{-}(z, \bar{z}) .
$$

So in all these cases, we can use our free field realization to construct indecomposable but reducible modules. In the case of $m=0$, we have both highest and lowest weight modules. These modules now deserve further investigation. We plan to study the combined left-right action of currents on these modules in the near future. The results then have to be compared to the semi-typicals of our harmonic analysis. Finally, let us note that there is a third free field realization simply by replacing $\tilde{Y}(z)$ and $\partial X(z)$ by a $\beta \gamma$ bosonic ghost algebra. This one will also be investigated later.

\section{Conclusion}

This work together with [4] puts one in the situation to study conformal field theories with chiral algebra the affinization of $\widetilde{\mathfrak{g l}(1 \mid 1)}$. We have studied the harmonic analysis on the supergroup in detail and especially we have found that all irreducible modules are part of larger indecomposable but reducible modules. We then have found two free field realizations for the associated CFT. The next question is to understand the spectrum of the full CFT better. For this, one needs to study the free field modules in detail. Especially the structure of semi-typicals and atypicals from the symplectic fermion free field realization needs to be worked out. In logarithmic CFT, indecomposable structure of modules is related to logarithmic singularities in correlation functions. Screening charges provide integral formula for correlation functions, so that one can use our findings to compute those. Since all our modules in the harmonic analysis are submodules of indecomposable but reducible ones we expect that logarithmic singularities will be a generic feature. This will be in strong contrast to logarithmic CFTs based on non Takiff supergroups.

Our findings have the drawback, that the Takiff superalgebra forced us to allow for polynomials in the variable $\tilde{y}$. In the conformal field theory this suggests to also allow for polynomials 
in the field $\tilde{y}$. This is unusual, as, e.g., the free boson CFT does not allow for fields of analogous type. Of course the OPE involving such polynomials will automatically yield logarithmic singularities. As mentioned in the end of last section, a possible way out is to replace $\tilde{Y}$ and $\partial Y$ by a $\beta \gamma$ bosonic ghost algebra. In that case we would have no semi-typical modules but still typical and atypical ones.

There are many future directions as boundary states and boundary correlation functions. These can surely be studied along the lines of their non-Takiff analouges $[12,18]$. Recall, that in that case also harmonic analysis was the important starting point. We are more interested in Takiff superalgebra CFTs based on more complicated algebras. It was shown that $\widehat{\mathfrak{g l}}(1 \mid 1)$ has many interesting simple current extensions [14, 15], including $\widehat{\mathfrak{s l}}(2 \mid 1)$ at levels 1 and $-1 / 2$. Characters of all these extensions turned out to be mock Jacobi forms for atypical modules [1]. It is probable that the Takiff superalgebra of $\widehat{\mathfrak{s l}}(2 \mid 1)$ appears in extensions of the $\widetilde{\mathfrak{g l}(1 \mid 1)} \mathrm{CFT}$, and interesting mock modular-like objects are expected to appear as characters of extended algebra modules.

It is also obvious that more realistic applications of Takiff affine algebras and superalgebras will require detailed understanding of Takiff affine $\widetilde{\mathfrak{s l}(2)}$. It seems natural to start its investigation from harmonic analysis, as a mini superspace toy model. One can expect here more involved structures of modules under the action of left- and right-invariant vector fields on the group manifold, since the representation theory of $\widetilde{\mathfrak{s l}(2)}$ consists of infinite-dimensional modules. It is also natural to expect that its free field realization will contain usual Wakimoto $\widehat{\mathfrak{s l}}(2)$ realization embedded into it.

\section{Acknowledgements}

A. Babichenko is thankful for hospitality to DESY Hamburg Theory Group and to ETH Zurich Institute for Theoretical Physics, where the final part of this work was done. His work was supported by SFB676, and his visit to ETH - by Pauli Center for Theoretical Studies. T. Creutzig is supported by NSERC Research Grant (Project \#: RES0020460).

\section{References}

[1] Alfes C., Creutzig T., The mock modular data of a family of superalgebras, Proc. Amer. Math. Soc. 142 (2014), 2265-2280, arXiv:1205.1518.

[2] Ashok S.K., Benichou R., Troost J., Asymptotic symmetries of string theory on $\mathrm{AdS}_{3} \times S^{3}$ with RamondRamond fluxes, J. High Energy Phys. 2009 (2009), no. 10, 051, 31 pages, arXiv:0907.1242.

[3] Ashok S.K., Benichou R., Troost J., Conformal current algebra in two dimensions, J. High Energy Phys. 2009 (2009), no. 6, 017, 35 pages, arXiv:0903.4277.

[4] Babichenko A., Ridout D., Takiff superalgebras and conformal field theory, J. Phys. A: Math. Theor. 46 (2013), 125204, 26 pages, arXiv:1210.7094.

[5] Benichou R., Troost J., The conformal current algebra on supergroups with applications to the spectrum and integrability, J. High Energy Phys. 2010 (2010), no. 4, 121, 47 pages, arXiv:1002.3712.

[6] Candu C., Creutzig T., Mitev V., Schomerus V., Cohomological reduction of sigma models, J. High Energy Phys. 2010 (2010), no. 5, 047, 39 pages, arXiv:1001.1344.

[7] Candu C., Mitev V., Quella T., Saleur H., Schomerus V., The sigma model on complex projective superspaces, J. High Energy Phys. 2010 (2010), no. 2, 015, 48 pages, arXiv:0908.0878.

[8] Creutzig T., Branes in supergroups, arXiv:0908.1816.

[9] Creutzig T., Geometry of branes on supergroups, Nuclear Phys. B 812 (2009), 301-321, arXiv:0809.0468.

[10] Creutzig T., Hikida Y., Branes in the OSP(1|2) WZNW model, Nuclear Phys. B 842 (2011), 172-224, arXiv:1004.1977. 
[11] Creutzig T., Hikida Y., Rønne P.B., Supergroup - extended super Liouville correspondence, J. High Energy Phys. 2011 (2011), no. 6, 063, 26 pages, arXiv:1103.5753.

[12] Creutzig T., Quella T., Schomerus V., Branes in the GL(1|1) WZNW model, Nuclear Phys. B 792 (2008), 257-283, arXiv:0708.0583.

[13] Creutzig T., Ridout D., Logarithmic conformal field theory: beyond an introduction, J. Phys. A: Math. Theor. 46 (2013), 494006, 72 pages, arXiv:1303.0847.

[14] Creutzig T., Ridout D., Relating the archetypes of logarithmic conformal field theory, Nuclear Phys. B 872 (2013), 348-391, arXiv:1107.2135.

[15] Creutzig T., Ridout D., W-algebras extending $\widehat{\mathfrak{g l}}(1 \mid 1)$, in Lie Theory and its Applications in Physics, Springer Proc. Math. Stat., Vol. 36, Springer, Tokyo, 2013, 349-367, arXiv:1111.5049.

[16] Creutzig T., Rønne P.B., The GL(1|1)-symplectic fermion correspondence, Nuclear Phys. B 815 (2009), 95-124, arXiv:0812.2835.

[17] Creutzig T., Rønne P.B., From world-sheet supersymmetry to super target spaces, J. High Energy Phys. 2010 (2010), no. 11, 021, 31 pages, arXiv:1006.5874.

[18] Creutzig T., Schomerus V., Boundary correlators in supergroup WZNW models, Nuclear Phys. B 807 (2009), 471-494, arXiv:0804.3469.

[19] Figueroa-O'Farrill J.M., Stanciu S., Nonsemisimple Sugawara construction, Phys. Lett. B 327 (1994), 40-46, hep-th/9402035.

[20] Figueroa-O'Farrill J.M., Stanciu S., Nonreductive WZW models and their CFTs, Nuclear Phys. B 458 (1996), 137-164, hep-th/9506151.

[21] Flohr M.A.I., Bits and pieces in logarithmic conformal field theory, Internat. J. Modern Phys. A 18 (2003), 4497-4591, hep-th/0111228.

[22] Frenkel I.B., Zhu Y., Vertex operator algebras associated to representations of affine and Virasoro algebras, Duke Math. J. 66 (1992), 123-168.

[23] Gaberdiel M.R., An algebraic approach to logarithmic conformal field theory, Internat. J. Modern Phys. A 18 (2003), 4593-4638, hep-th/0111260.

[24] Gainutdinov A.M., Jacobsen J.L., Saleur H., Vasseur R., A physical approach to the classification of indecomposable Virasoro representations from the blob algebra, Nuclear Phys. B 873 (2013), 614-681, arXiv:1212.0093.

[25] Gainutdinov A.M., Read N., Saleur H., Associative algebraic approach to logarithmic CFT in the bulk: the continuum limit of the $\mathfrak{g l}(1 \mid 1)$ periodic spin chain, Howe duality and the interchiral algebra, arXiv:1207.6334.

[26] Gainutdinov A.M., Read N., Saleur H., Continuum limit and symmetries of the periodic gl(1|1) spin chain, Nuclear Phys. B $\mathbf{8 7 1}$ (2013), 245-288, arXiv:1112.3403.

[27] Geoffriau F., Sur le centre de l'algèbre enveloppante d'une algèbre de Takiff, Ann. Math. Blaise Pascal 1 (1994), 15-31.

[28] Götz G., Quella T., Schomerus V., The WZNW model on PSU(1,1|2), J. High Energy Phys. 2007 (2007), no. 3, 003, 48 pages, hep-th/0610070.

[29] Guruswamy S., LeClair A., Ludwig A.W.W., $\mathfrak{g l}(N \mid N)$ super-current algebras for disordered Dirac fermions in two dimensions, Nuclear Phys. B 583 (2000), 475-512, cond-mat/9909143.

[30] Hikida Y., Schomerus V., Structure constants of the OSp(1|2) WZNW model, J. High Energy Phys. 2007 (2007), no. 12, 100, 32 pages, arXiv:0711.0338.

[31] Kausch H.G., Symplectic fermions, Nuclear Phys. B 583 (2000), 513-541, hep-th/0003029.

[32] LeClair A., The $\mathfrak{g l}(1 \mid 1)$ super-current algebra: the rôle of twist and logarithmic fields, Adv. Theor. Math. Phys. 13 (2009), 259-291, arXiv:0710.2906.

[33] Mathieu P., Ridout D., From percolation to logarithmic conformal field theory, Phys. Lett. B 657 (2007), 120-129, arXiv:0708.0802.

[34] Mitev V., Quella T., Schomerus V., Principal chiral model on superspheres, J. High Energy Phys. 2008 (2008), no. 11, 086, 45 pages, arXiv:0809.1046.

[35] Mitev V., Quella T., Schomerus V., Conformal superspace $\sigma$-models, J. Geom. Phys. 61 (2011), 1703-1716, arXiv:1210.8159.

[36] Mohammedi N., Wess-Zumino-Novikov-Witten models based on Lie superalgebras, Phys. Lett. B 331 (1994), 93-98, hep-th/9404132. 
[37] Pearce P.A., Rasmussen J., Zuber J.-B., Logarithmic minimal models, J. Stat. Mech. Theory Exp. 2006 (2006), P11017, 36 pages, hep-th/0607232.

[38] Piroux G., Ruelle P., Logarithmic scaling for height variables in the abelian sandpile model, Phys. Lett. B 607 (2005), 188-196, cond-mat/0410253.

[39] Quella T., Schomerus V., Free fermion resolution of supergroup WZNW models, J. High Energy Phys. 2007 (2007), no. 9, 085, 51 pages, arXiv:0706.0744.

[40] Quella T., Schomerus V., Creutzig T., Boundary spectra in superspace $\sigma$-models, J. High Energy Phys. 2008 (2008), no. 10, 024, 26 pages, arXiv:0712.3549.

[41] Read N., Saleur H., Exact spectra of conformal supersymmetric nonlinear sigma models in two dimensions, Nuclear Phys. B 613 (2001), 409-444, hep-th/0106124.

[42] Read N., Saleur H., Associative-algebraic approach to logarithmic conformal field theories, Nuclear Phys. B 777 (2007), 316-351, hep-th/0701117.

[43] Rozansky L., Saleur H., $S$ - and $T$-matrices for the super U(1,1) WZW model. Application to surgery and 3-manifolds invariants based on the Alexander-Conway polynomial, Nuclear Phys. B 389 (1993), 365-423, hep-th/9203069.

[44] Saleur H., Schomerus V., On the SU(2|1) WZNW model and its statistical mechanics applications, Nuclear Phys. B 775 (2007), 312-340, hep-th/0611147.

[45] Schomerus V., Saleur H., The GL(1|1) WZW-model: from supergeometry to logarithmic CFT, Nuclear Phys. B $\mathbf{7 3 4}$ (2006), 221-245, hep-th/0510032.

[46] Takiff S.J., Rings of invariant polynomials for a class of Lie algebras, Trans. Amer. Math. Soc. 160 (1971), 249-262.

[47] Vasseur R., Jacobsen J.L., Saleur H., Logarithmic observables in critical percolation, J. Stat. Mech. Theory Exp. 2012 (2012), L07001, 11 pages, arXiv:1206.2312.

[48] Wilson B.J., A character formula for the category $\tilde{\mathcal{O}}$ of modules for affine $\mathfrak{s l}(2)$, Int. Math. Res. Not. 2008 (2008), Art. ID rnn 092, 29 pages, arXiv:0711.0727.

[49] Wilson B.J., Highest-weight theory for truncated current Lie algebras, J. Algebra 336 (2011), 1-27, arXiv:0705.1203.

[50] Zirnbauer M.R., Conformal field theory of the integer quantum Hall plateau transition, hep-th/9905054. 